\title{
Sampling From a System-Theoretic Viewpoint: Part I-Concepts and Tools
}

\author{
Gjerrit Meinsma and Leonid Mirkin, Member, IEEE
}

\begin{abstract}
This paper is first in a series of papers studying a system-theoretic approach to the problem of reconstructing an analog signal from its samples. The idea, borrowed from earlier treatments in the control literature, is to address the problem as a hybrid model-matching problem in which performance is measured by system norms. In this paper we present the paradigm and revise underlying technical tools, such as the lifting technique and some topics of the operator theory. This material facilitates a systematic and unified treatment of a wide range of sampling and reconstruction problems, recovering many hitherto considered different solutions and leading to new results. Some of these applications are discussed in the second part.
\end{abstract}

Index Terms-Causality, lifting, sampling and reconstruction, signal modeling, stability, system norms.

\section{INTRODUCTION}

$\mathbf{T}$ HE problem of reconstructing a continuous-time signal from its sampled measurements may be, perhaps simplistically, described by the block-diagram in Fig. 1. Here $y$ is a continuous-time signal, which is sampled by an A/D converter (sampler) $\mathcal{S}$, the resulting discrete-time signal $\bar{y}$ is processed by a digital filter $\overline{\mathcal{F}}$, and the output of the latter, $\bar{u}$, is converted back to continuous time by a D/A converter (hold) $\mathcal{H}$. Throughout, we refer to the (continuous-time) system from $y$ to $u$ as the hybrid signal processor (HSP) and denote it $\mathcal{F}_{\mathrm{HSP}}$.

Our goal typically is to generate $u$ as close to $y$ as possible. Sampling/reconstruction (SR) problems of this kind are important in numerous signal and image processing and control applications and have been extensively studied in both mathematical and engineering literature, see [1]-[5] for detailed overviews of the subject and a comprehensive bibliography. Classical studies are mainly concerned with the conditions under which perfect reconstruction of $y$ is possible and the choice of the corresponding hold (interpolator) $\mathcal{H}$. This leads to the celebrated Sampling Theorem and its generalizations [1], [3], [5]. Such approaches, however, rely upon assumptions that are seldom realistic (e.g., require $y$ to be bandlimited or

Manuscript received November 02, 2009; accepted March 21, 2010. Date of publication April 05, 2010; date of current version June 16, 2010. The associate editor coordinating the review of this manuscript and approving it for publication was Prof. Haldun M. Ozaktas. This work was supported by the Israel Science Foundation by Grant 1238/08.

G. Meinsma is with the Department of Applied Mathematics, University of Twente, 7500 AE Enschede, The Netherlands (e-mail: g.meinsma@utwente.nl).

L. Mirkin is with the Faculty of Mechanical Engineering, Technion-IIT,

Haifa 32000, Israel (e-mail: mirkin @technion.ac.il).

Color versions of one or more of the figures in this paper are available online at http://ieeexplore.ieee.org.

Digital Object Identifier 10.1109/TSP.2010.2047641

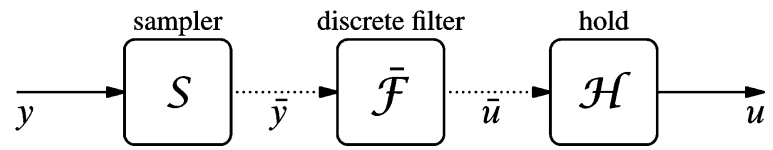

Fig. 1. Hybrid signal processor (HSP) $\mathcal{F}_{\text {HSP }}$.

generated by a discrete sequence), and result in interpolators that might be hard to implement or approximate.

These considerations prompted more recent studies to give up on the perfect reconstruction requirement. An example of such a setup is the reconstruction in shift-invariant spaces [2], [4], where $\overline{\mathcal{F}}$ is designed, for fixed sampling and hold circuits, to satisfy some weaker requirements. Examples of these requirements are the consistency [2], which is the perfect reconstruction of samples $\bar{y}$, or the (dual, in a sense) minimization of the error restricted to the image of $\mathcal{H}$ [6]. An advantage here is the full control over properties of $\mathcal{S}$ and $\mathcal{H}$, which may be chosen to simplify their implementation (like splines) and approximation (like truncating to impose causality constraints). This choice, however, might not be justifiable performance-wise. Moreover, the design of $\overline{\mathcal{F}}$ accounts only for a part of the reconstruction error rather than the analog error itself.

Direct optimization of analog error signals is the core of the sampled-data control theory [7], [8], which studies digital control of analog systems. Motivated by this, [9] proposed to cast SR problems as a hybrid $H^{\infty}$-causal minmax - model-matching setup (the idea can be traced back to [10] and [11]). This is a special case of the standard sampled-data control problem and can therefore be handled by available control methods, adopted to the relaxation of the causality of $\overline{\mathcal{F}}$. Advantages of this approach are that it explicitly addresses the analog error and does not restrict the class of input signals. The method of [9], however, is based on several intermediate transformations, which blur the structure of the solution. In fact, no closed-form formulae for this approach exist. Moreover, the design methodology adopted there is also limited to the case when both $\mathcal{S}$ and $\mathcal{H}$ are fixed.

Excluding the acquisition and reconstruction devices from the design cycle, which limits the achievable reconstruction performance, is not always justifiable. Technological constraints, which restrict the complexity of A/D and D/A circuits, become less severe taking into account the progress in hardware technology. Other constraints might merely result from limitation of existing design methods. For example, the decay rate of the interpolating kernel is considered an important factor in the choice of $\mathcal{H}$ [2]. Yet this appears to be brought about by the need to truncate it afterwards in order to impose causality constraints on the 
reconstructor. If these constraints were explicitly accounted for in the design stage, the kernel decay would not be so important.

This series of papers aims at developing a systematic approach to the design of SRs, in which sampling and/or hold devices can be incorporated into the design process, that allows to impose causality constraints as part of the design, and which is comprehensive, covering many known problems as special cases. Towards this end, we adopt the system-theoretic viewpoint, by which signals are modeled by systems and reconstruction performance is measured by system norms. The system-theoretic approach enables us to treat signals of different physical nature and properties (e.g., stochastic and deterministic) in a unified manner.

The goal of this paper is to present underlying concepts and the technical material required for the system-theoretic analysis of SR problems. In particular, we place the main emphasis on the lifting, which is our main analysis tool and may be thought of as an analog counterpart of the common polyphase decomposition [12]. Although many of the results presented here are not new, we believe that their compact and unified exposition is of its own tutorial value. Moreover, this material can be found mainly in the control literature, where systems are assumed to be causal and hence are considered on the semi axes $\mathbb{R}^{+}$and $\mathbb{Z}^{+}$ only. In signal processing applications noncausal and relaxedly causal systems are important, so we have to deal with systems on the whole time axes $\mathbb{R}$ and $\mathbb{Z}$, which calls for certain, sometimes nontrivial, modifications to be made. Also, the lifting is predominantly studied in the state-space setting in the control literature, while we emphasize here realization-free input/output relations, such as convolutions and infinite-dimensional transfer functions. This is pivotal in Part II, where optimal solutions do not have realizations. Last but not least, we do present new results, like the Key Lifting Formula (Theorem 4.1) and the frequency-domain characterization of the relaxed causality (Theorem 6.2).

The paper is organized as follows. In Section II we introduce a general optimization setup, the study of which is the leitmotif of this series of papers. Section III presents the lifting technique and collects some time-domain facts and definitions. In Section IV some frequency-domain lifting definitions and results are presented. Spaces of signals and systems in the lifted domain and corresponding metrics are considered in Section V. Finally, Section VI presents the notions of stability and causality and their frequency-domain characterizations.

\section{Notation}

Throughout, $h$ denotes the sampling period and $\omega_{N}:=\pi / h$ is the associated Nyquist frequency. The sinc function with knots at multiples of $h$ is $\operatorname{sinc}_{h}(t):=\sin \left(\omega_{N} t\right) /\left(\omega_{N} t\right)$. Signals are represented by lowercase symbols such as $y(t): \mathbb{R} \rightarrow \mathbb{C}$ and overbars indicate discrete time signals, $\bar{y}[k]: \mathbb{Z} \rightarrow \mathbb{C}$. For any set $A$ the indicator function $\mathbb{1}_{A}(t)$ is 1 if $t \in A$ and is zero elsewhere. The unit step (which is actually $\mathbb{1}_{\mathbb{R}^{+}}(t)$ ) is denoted $\mathbb{1}(t)$ (in continuous time) and $\overline{\mathbb{1}}[k]$ in discrete time. Similarly $\delta(t)$ is the Dirac delta function (understood implicitly as the causal $\delta\left(t-0^{+}\right)$) and $\bar{\delta}[k]$ is the discrete unit pulse. The number of elements of a vector-valued signal $v$ is denoted by $n_{v}$.

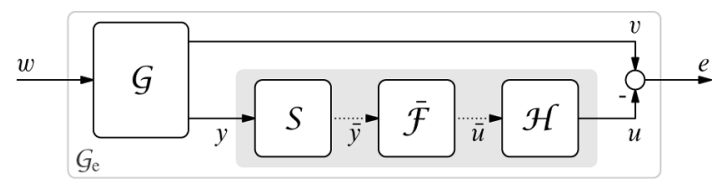

Fig. 2. Sampling/reconstruction (SR) setup.

Uppercase calligraphic symbols, like $\mathcal{G}$, denote continuoustime systems in time domains, the impulse response/kernel of which is denoted with lowercase symbols, such as $g$, and the corresponding transfer function/frequency response is presented by uppercase symbols, like $G(s)$ and $G(\mathrm{j} \omega)$. Discrete-time systems, kernels, etcetera are denoted by overbars, like $\overline{\mathcal{G}}, \bar{g}$, etc. Other more specific notation for lifted signals and systems is defined later (in particular, see Remark 3.1).

By $\mathbb{Z}_{l}^{+}\left(\mathbb{Z}_{l}^{-}\right)$we denote the set of all integers larger or equal to (smaller than) $l$. The symbols $\mathbb{T}, \mathbb{D}$, and $\overline{\mathbb{D}}$ stand for the unit circle $(|z|=1)$, the open unit disk $(|z|<1)$, and the closed unit disk $(|z| \leq 1)$ in the complex plane, respectively.

$L_{\mathbb{B}}^{2}(\mathbb{A})$ is the set of functions $f: \mathbb{A} \rightarrow \mathbb{B}$ that have finite norm $\|f\|_{2}:=\left(\int_{t \in A}\|f(t)\|_{\mathbb{B}}^{2} \mathrm{~d} t\right)^{1 / 2}$, where $\|\cdot\|_{\mathbb{B}}$ denotes some given norm on $\mathbb{B}$ (in case $\mathbb{B}=\mathbb{C}^{n_{f}}$ we assume the standard Euclidean norm $|\cdot|)$. Sometimes we use the notation $\mathbb{L}:=$ $L^{2} \mathbb{C}[0, h)$. The space $\ell_{\mathbb{B}}^{2}(\mathbb{Z})$ is the set of $\bar{f}: \mathbb{Z} \rightarrow \mathbb{B}$ with finite norm $\|\bar{f}\|_{2}:=\left(\sum_{k \in \mathbb{Z}}\|\bar{f}[k]\|_{\mathbb{B}}^{2}\right)^{1 / 2}$. Some (or all) space arguments in the notation for $L^{2}$ and $\ell^{2}$ will be dropped when they are irrelevant or clear from the context.

\section{SETUP}

In this series of papers, we study the SR setup shown in Fig. 2. Here $v$ is an (unknown) analog signal, which is to be reconstructed from sampled measurements of a related analog signal $y$. Both $v$ and $y$ are modeled as outputs of a continuous-time system $\mathcal{G}$ (signal generator) driven by a common input $w$ with known characteristics. The signal $u$ is the reconstruction of $v$ on the basis of $y$. This signal $u$ is the output of the HSP, which is highlighted by the dark shadowed box in Fig. 2. It includes a sampler $\mathcal{S}$, a digital filter $\overline{\mathcal{F}}$, and a reconstructor, or hold, $\mathcal{H}$ (for more details see Section II-B below). Our goal then is to design an HSP (or only some of its components) to minimize a "size" (norm) of the error system $\mathcal{G}_{e}$ (the light shadowed box in Fig. 2) which is the mapping from $w$ to the reconstruction error $e:=v-u$. Minimization of the mapping enforces that the output $u$ of the HSP is in a sense optimally close to the signal $v$ that we intend to reconstruct. This renders the optimal SR problem a systems optimization problem.

\section{A. Paradigms}

Two central aspects of the system-theoretic formulation of SR problems are the use of the signal generator $\mathcal{G}$ to model signals and the use of system norms to measure the SR performance. These aspects, which have have proved useful in control applications, are possibly somewhat latent in the SR literature, so we start with a brief exposition of the underlying ideas. 
1) Signal Generator: Clearly, the reconstruction of a signal $v$ on the basis of $y$ makes sense only if the two signals share certain qualities. To model cross-correlations, dynamic relations, etcetera between $v$ and $y$, one may choose to consider both $v$ and $y$ as the outcome of a (possibly fictitious) signal generator $\mathcal{G}$ driven by a common signal $w$ having known and normalized features (such as being white or belonging to some bounded set). Below we indicate how these goals can be attained. To this end, partition the signal generator $\mathcal{G}$ compatible with the signal partition in Fig. 2 as

$$
\mathcal{G}=\left[\begin{array}{l}
\mathcal{G}_{v} \\
\mathcal{G}_{y}
\end{array}\right]
$$

The simplest choice of its components would be $\mathcal{G}_{v}=\mathcal{G}_{y}=I$, which reflects the assumptions that $v=y$ and that $v$ is the only exogenous input. If the measured signal passes through an antialiasing filter $\mathcal{F}_{a}$, we should pick $\mathcal{G}_{y}=\mathcal{F}_{a}$ instead. If the measurement of $v$ is corrupted by a measurement noise, $n$, the latter has to be included into the exogenous signal, so that $w=\left[\begin{array}{l}v \\ n\end{array}\right]$ and we end up with $\mathcal{G}_{v}=\left[\begin{array}{ll}I & 0\end{array}\right]$ and $\mathcal{G}_{y}=\left[\begin{array}{ll}I & I\end{array}\right]$ (or $\mathcal{G}_{y}=\mathcal{F}_{a}\left[\begin{array}{ll}I & I\end{array}\right]$, if an antialiasing filter is present). If the velocity of $y$ should be reconstructed, we choose $\mathcal{G}_{v}=\mathcal{F}_{d}$, where $\mathcal{F}_{d}$ is the differentiator, having the frequency response $F_{d}(j \omega)=\mathrm{j} \omega$. Thus, the problem of reconstructing the velocity from filtered noisy position measurements is formalized via as$\operatorname{signing} \mathcal{G}_{v}=\left[\begin{array}{ll}\mathcal{F}_{d} & 0\end{array}\right], \mathcal{G}_{y}=\mathcal{F}_{a}\left[\begin{array}{ll}I & I\end{array}\right]$, and $w=\left[\begin{array}{l}x \\ n\end{array}\right]$, where $x$ is the position.

In these examples, the exogenous input $w$ still consists of a combination of "real" signals such as position and noise, each with its own dynamical properties and physical domain/unit. To simplify their joint treatment, they can be modeled in terms of some normalized signal having favorable mathematical properties, passing through known systems. For example, if the signal to be reconstructed, $v$, is slow, it can be modeled as $v=\mathcal{F}_{v} w_{v}$, where $\mathcal{F}_{v}$ is a low-pass filter and $w_{v}$ is some fictitious normalized signal. Examples of such signals are white noise in the stochastic case ${ }^{1}$ and the $\delta$-impulse in the deterministic case, both of which have normalized flat spectra. A fast measurement noise, $n$, can then be modeled via another normalized signal, $w_{n}$, as $n=\mathcal{F}_{n} w_{n}$ for some high-pass filter $\mathcal{F}_{n}$. In this case, the problem of reconstructing a signal from filtered noisy measurements can be formalized via $\mathcal{G}_{v}=\left[\begin{array}{ll}\mathcal{F}_{v} & 0\end{array}\right]$ and $\mathcal{G}_{y}=\mathcal{F}_{a}\left[\mathcal{F}_{v} \quad \mathcal{F}_{n}\right]$. The exogenous signal, $w=\left[\begin{array}{c}w_{v} \\ w_{n}\end{array}\right]$, is then a fictitious normalized signal all components of which are on an equal footing and have similar properties; all structural properties are represented by $\mathcal{G}$.

Remark 2.1: The use of modeling filters, like $\mathcal{F}_{v}$ and $\mathcal{F}_{n}$ above, does not necessarily intend to constrain signals (e.g., $v$ and $n$ ) to belong to a (finite-dimensional) subspace of the space of continuous-time signals, like those discussed in [3]. In many cases these filters may be thought of as functions, reshaping the metric used to measure the SR performance. Through the choice of these filters we thus just emphasize certain aspects of signal properties, like their dominant frequency bands.

\footnotetext{
${ }^{1}$ In this case $F_{v}(\mathrm{j} \omega)\left[F_{v}(\mathrm{j} \omega)\right]^{*}$ is actually the spectral density of $v$.
}

2) Performance Measures: The normalization of the exogenous input $w$ makes it possible to express the size of the reconstruction error signal $e$ in terms of the size of the error system $\mathcal{G}_{e}$ mapping $w$ to $e$. We use two measures of the size of $\mathcal{G}_{e}$ : its $L^{2}$ and $L^{\infty}$ norms. Below we briefly discuss these formalisms. To avoid the introduction of involved technicalities at this stage, we assume for the moment that $\mathcal{G}_{e}$ is time invariant. Although this is practically never the case for the hybrid system in Fig. 2, extensions are conceptually straightforward (they are discussed in Section V).

The Hilbert space $L^{2}{ }_{p \times m}(\mathrm{j} \mathbb{R})$, or simply $L^{2}(\mathrm{j} \mathbb{R})$ when the dimensions are irrelevant or clear from the context, is the set of functions $F(s): \mathrm{j} \mathbb{R} \rightarrow \mathbb{C}^{p \times m}$ for which

$$
\|F\|_{2}:=\left(\frac{1}{2 \pi} \int_{-\infty}^{\infty}\|F(\mathrm{j} \omega)\|_{F}^{2} \mathrm{~d} \omega\right)^{1 / 2}<\infty
$$

where $\|\cdot\|_{F}$ is the Frobenius matrix norm. The quantity $\|F\|_{2}$ is called the $L^{2}$-norm of $F(s)$. If $F(s)$ is the transfer function of an LTI system $\mathcal{F}$, we also refer to this quantity as the $L^{2}$-norm of $\mathcal{F}$ and denote it as $\|\mathcal{F}\|_{2}$. This norm has clear interpretations, both deterministic and stochastic, in terms of the input and output signals of $\mathcal{F}$. In the deterministic setting, it is readily seen from the Parseval's equality that $\|\mathcal{F}\|_{2}^{2}$ is the sum of the energies of the responses of $\mathcal{F}$ to $\delta$-impulses applied at each of its $m$ input components. In the stochastic setting, $\|\mathcal{F}\|_{2}^{2}$ is the power, that is, the sum of the variances of the $p$ output components of $\mathcal{F}$ in the case when the input is a zero-mean unit intensity white noise process [13, Sec. 3.8].

The space $L_{p \times m}^{\infty}(\mathrm{j} \mathbb{R})$, or simply $L^{\infty}(\mathrm{j} \mathbb{R})$, is the set of functions $F(s): \mathrm{j} \mathbb{R} \rightarrow \mathbb{C}^{p \times m}$, the $L^{\infty}$-norm of which

$$
\|F\|_{\infty}:=\operatorname{ess}_{\omega \in \mathbb{R}} \sup _{\max }[F(\mathrm{j} \omega)]<\infty .
$$

Similarly to the $L^{2}$ case, if $F(s)$ is the transfer function of an LTI system $\mathcal{F}$, the quantity defined by (2) is referred to as the $L^{\infty}$-norm of $\mathcal{F}$ and denoted by $\|\mathcal{F}\|_{\infty}$. This norm can also be interpreted in terms of signals: $\|\mathcal{F}\|_{\infty}^{2}$ is the maximal energy of the output over all inputs of unit energy [14, Thm. A.6.26], i.e., the maximal energy gain of $\mathcal{F}$.

Returning to the setup in Fig. 2, the minimization of $\left\|\mathcal{G}_{e}\right\|_{2}$ in the stochastic case corresponds to (average) power or mean square minimization of the continuous-time reconstruction error $e$ (energy minimization in the deterministic case). Thus, this is merely a hybrid version of the classical Wiener (or Kalman) filtering problem [15]. The minimization of $\left\|\mathcal{G}_{e}\right\|_{\infty}$ corresponds to the minmax formulation, in which the mean-square error is minimized for a worst-case input of unit energy. In fact, the $L^{2}$ and $L^{\infty}$ approaches represent two extremes in our assumptions about the exogeneous signals. The former assumes that these signals are completely known, whereas the latter-that they are completely unknown, other than having finite power or energy. The "gray areas" in between may then be (implicitly) covered by the use of weighting filters.

Remark 2.2: It is not hard to imagine situations where some of the exogenous inputs are known and some are not. This might call for the use of mixed $L^{2} / L^{\infty}$ strategies, such as minimizing the $L^{2}$-norm of a subsystem of $\mathcal{G}_{e}$ while keeping the $L^{\infty}$-norm of the other subsystem below some prescribed level [16]. Such 
problems, however, result in complicated solutions that lack the structure and transparency of their pure $L^{2}$ and $L^{\infty}$ counterparts. We, therefore, do not pursue this line here. After all, it is rarely possible to squeeze all requirements into a single optimization problem, so that the optimization in engineering should be considered as merely a tool to achieve meaningful and transparent solutions rather than a goal per se.

The expression of the performance requirements via system norms simplifies the treatment of deterministic and stochastic signals via a unified formalism and brings some other (conceptual) advantages. For example, the $L^{\infty}$ formulation is well suited for the sake of shaping the spectrum of the reconstruction error. To see this, consider the noise-free scalar setting and let $v$ be modeled as $v=\mathcal{F}_{v} w$. Then

$$
\left\|\mathcal{G}_{e}\right\|_{\infty}<1 \Rightarrow|e(\mathrm{j} \omega)|<\frac{1}{\left|F_{v}(\mathrm{j} \omega)\right|}|v(\mathrm{j} \omega)|, \quad \forall \omega \in \mathbb{R} .
$$

Thus, a desired shape of the error spectrum can be pursued via an appropriate choice of $\mathcal{F}_{v}$. The existence of a reconstructor guaranteeing $\left\|\mathcal{G}_{e}\right\|_{\infty}<1$, which is the question that can be conclusively answered, is then the success indicator. Another advantage of the system-based treatment is a (relative) simplicity with which causality constraints can be imposed upon the reconstructor (see Section VI).

\section{B. Components}

We now detail some of the components of the configuration in Fig. 2. In particular, below we address the HSP, containing a sampler, a discrete filter and a hold.

1) Sampler: By a sampling device $\mathcal{S}$ we understand any linear device transforming a function $y(t): \mathbb{R} \rightarrow \mathbb{C}^{n_{y}}$ into a function $\bar{y}[k]: \mathbb{Z} \rightarrow \mathbb{C}^{n_{\bar{y}}}$. Assuming that

$$
\mathcal{S}(y(\cdot-h))=(\mathcal{S} y)[\cdot-1]
$$

which can be thought of as A/D shift invariance, a general model for such a device is

$$
\bar{y}=\mathcal{S} y: \quad \bar{y}[k]=\int_{-\infty}^{\infty} \psi(k h-s) y(s) \mathrm{d} s, \quad k \in \mathbb{Z}
$$

for some $\psi(t)$, called the sampling function. The ideal sampler $\mathcal{S}_{\text {Idl }}$, generating $\bar{y}[k]=y(k h)$ and well defined for continuous inputs, has $\psi(t)=\delta(t)$. The continuity of $y$ can be ensured by an antialiasing filter $\mathcal{F}_{a}$ having the impulse response $f_{a}(t)$. Such a filter can always be incorporated into $\mathcal{S}$, resulting in a sampler with $\psi(t)=f_{a}(t)$. In fact, a general sampler of the form (3) can always be presented as the cascade of an LTI system with the impulse response $\psi(t)$ and the ideal sampler. An important example, especially for the developments in Part II, [17], is the sinc-sampler, $\mathcal{S}_{\text {sinc }}$, having the sampling function $\psi_{\text {sinc }}(t):=(1 / h) \operatorname{sinc}_{h}(t)$. It can be viewed as the ideal lowpass filter with the cutoff frequency $\omega_{N}$ followed by the ideal sampler. Another example is the causal averaging sampler $\mathcal{S}_{A v}$, which corresponds to $\psi(t)=(1 / h) \mathbb{1}_{[0, h)}(t)$.

2) Hold: By a hold device $\mathcal{H}$ we understand a linear device transforming a function $\bar{u}[k]: \mathbb{Z} \rightarrow \mathbb{C}^{n_{\bar{u}}}$ into a function $u(t)$ : $\mathbb{R} \rightarrow \mathbb{C}^{n_{u}}$. Assuming D/A shift invariance, understood as

$$
\mathcal{H}(\bar{u}[\cdot-1])=(\mathcal{H} \bar{u})(\cdot-h)
$$

a general model of this device is

$$
u=\mathcal{H} \bar{u}: \quad u(t)=\sum_{i \in \mathbb{Z}} \phi(t-i h) \bar{u}[i], \quad t \in \mathbb{R}
$$

for some hold function ${ }^{2} \phi(t)$. The hold function is the response of $\mathcal{H}$ to the discrete unit pulse $\bar{\delta}[i]$. The hold can also be thought of as a modulator of the input sequence $\{\bar{u}[i]\}$. The standard zero-order hold $\mathcal{H}_{\mathrm{ZOH}}$, which keeps $u(t)$ constant over the intersample period, corresponds in this setting to $\phi(t)=\mathbb{1}_{[0, h)}(t)$. The predictive first-order hold $\mathcal{H}_{\mathrm{FOH}}$, which is a linear interpolator of two successive input values, has the "tent" hold function $\phi(t)=(1-|t| / h) \mathbb{1}_{[-h, h)}(t)$. It is readily seen that both these hold devices can be presented as the cascade of the impulsetrain modulator $\mathcal{H}_{\mathrm{ITM}}$, having the hold function $\phi(t)=\delta(t)$, and continuous-time LTI systems with the transfer functions $1-\mathrm{e}^{-s h} / s$ (for $\mathcal{H}_{\mathrm{ZOH}}$ ) and $\left(1-\mathrm{e}^{-s h} / s\right)^{2} \mathrm{e}^{s h}$ (for $\mathcal{H}_{\mathrm{FOH}}$ ). Another example of a hold device is the sinc-hold, $\mathcal{H}_{\text {sinc }}$, having the hold function $\phi_{\text {sinc }}(t):=\operatorname{sinc}_{h}(t)$. This is actually the interpolator from the Sampling Theorem.

Remark 2.3: We do not restrict the input and output dimension of $\mathcal{S}$ and $\mathcal{H}$. For example, the sampler may produce a vector-valued discrete signal $\left(n_{\bar{y}}>1\right)$ from a scalar analog signal $\left(n_{y}=1\right)$. This renders the setup general enough to describe multirate or nonuniform sampling problems (using the polyphase decomposition).

3) Discrete Part: A general form of the LTI discrete-time system $\overline{\mathcal{F}}$ is the convolution model

$$
\bar{u}=\overline{\mathcal{F}} \bar{y}: \quad \bar{u}[k]=\sum_{i \in \mathbb{Z}} \bar{f}[k-i] \bar{y}[i], \quad k \in \mathbb{Z}
$$

where the sequence $\bar{f}[k]$ is known as the impulse response of $\overline{\mathcal{F}}$. This system can always be absorbed into $\mathcal{S}$ or $\mathcal{H}$ via redefining the functions $\psi$ and $\phi$, respectively. When analyzing HSPs we thus may assume without loss of generality that $\overline{\mathcal{F}}=I$ or, equivalently, $\bar{f}[k]=\bar{\delta}[k]$. This assumption can also be made during the design if either sampler or hold (or both) is a design parameter. For implementation of HSPs it might however be advantageous to use a separate discrete filter.

\section{LIFTING IN TIME DOMAIN}

Let us return now to the HSP in Fig. 1 and consider it as a continuous-time system from $y$ to $u$. Assuming, without loss of generality, that $\overline{\mathcal{F}}=I$ and combining (3) and (4), we get

$$
\begin{aligned}
u(t) & =\sum_{i \in \mathbb{Z}} \phi(t-i h) \int_{-\infty}^{\infty} \psi(i h-s) y(s) \mathrm{d} s \\
& =\int_{-\infty}^{\infty} \sum_{i \in \mathbb{Z}} \phi(t-i h) \psi(i h-s) y(s) \mathrm{d} s .
\end{aligned}
$$

Thus, $\mathcal{F}_{\text {HSP }}$ is an integral operator of the form

$$
u(t)=\int_{-\infty}^{\infty} g(t, s) y(s) \mathrm{d} s
$$

with kernel

$$
g(t, s)=f_{\mathrm{HSP}}(t, s):=\sum_{i \in \mathbb{Z}} \phi(t-i h) \psi(i h-s) .
$$

\footnotetext{
${ }^{2}$ Thus, psi stands for sampler and phi for $h$ old.
} 


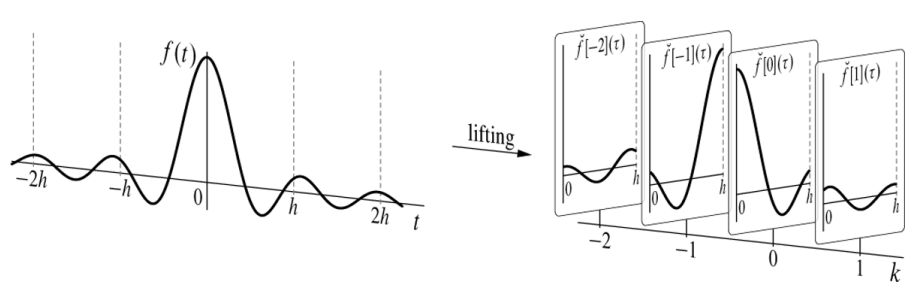

Fig. 3. Lifting analog signals (with $f(t)=\operatorname{sinc}_{0.44 h}(t)$ ).

System (6) is time invariant iff $g(t, s)=g(t+\sigma, s+\sigma)$ for all $\sigma \in \mathbb{R}$. This, in general, is not the case for the kernel $f_{\mathrm{HSP}}(t, s)$ above. Thus, operations of continuous time signals that include $\mathrm{A} / \mathrm{D}$ and $\mathrm{D} / \mathrm{A}$ converters are not a time-invariant operation in general. Many of the techniques that are available for LTI systems can therefore not be readily applied to $\mathcal{F}_{\text {HSP. }}$. The time invariance can, however, be regained on noticing that

$$
f_{\mathrm{HSP}}(t, s)=f_{\mathrm{HSP}}(t+k h, s+k h), \quad \forall k \in \mathbb{Z} .
$$

This property, known as $h$-shift invariance, enables one to convert $\mathcal{F}_{\text {HSP }}$ into an equivalent shift-invariant system using the linear transformation called lifting, see books [7], [8] for more details and bibliography.

The lifting transformation-or simply lifting — can be seen as a way of separating the behavior into a fully time invariant discrete-time behavior and a finite-horizon continuous-time (intersample) behavior. Fig. 3 explains the idea and the formal definition is given here.

Definition 3.1: For any signal $f: \mathbb{R} \rightarrow \mathbb{C}^{n_{f}}$, the lifting $\breve{f}: \mathbb{Z} \rightarrow\left\{[0, h) \rightarrow \mathbb{C}^{n_{f}}\right\}$ is the sequence of functions $\{\breve{f}[k]\}$ defined as

$$
\breve{f}[k](\tau)=f(k h+\tau), \quad k \in \mathbb{Z}, \quad \tau \in[0, h) .
$$

In other words, with lifting we consider a function on $\mathbb{R}$ as a sequence of functions on $[0, h)$. Clearly, this incurs no loss of information, it is merely another representation of the signal. The rationale behind this representation is to "forbid" any time shift but multiples of $h$. This implies that if a continuous-time system $u=\mathcal{G} y$ is $h$-shift invariant, then in lifted representation, $\breve{u}=\breve{\mathcal{G}} \breve{y}$, it is shift invariant.

More explicitly, let $\mathcal{G}$ be an $h$-shift-invariant system defined by (6). In the lifted domain this mapping reads

$$
\begin{aligned}
\breve{u}[k](\tau) & =u(k h+\tau)=\int_{-\infty}^{\infty} g(k h+\tau, \sigma) y(\sigma) \mathrm{d} \sigma \\
& =\sum_{i \in \mathbb{Z}} \int_{0}^{h} g(k h+\tau, i h+\sigma) y(i h+\sigma) \mathrm{d} \sigma \\
& =\sum_{i \in \mathbb{Z}} \int_{0}^{h} g((k-i) h+\tau, \sigma) \breve{y}[i](\sigma) \mathrm{d} \sigma
\end{aligned}
$$

which can be written as

$$
\breve{u}[k]=\sum_{i \in \mathbb{Z}} \breve{G}[k-i] \breve{y}[i]
$$

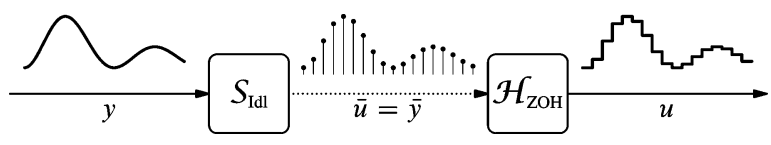

Fig. 4. Sample-and-hold circuit in the time domain.

where $\breve{G}[k], k \in \mathbb{Z}$, is the (lifted) impulse response system that maps functions on $[0, h)$ to functions on $[0, h)$ as

$$
\begin{aligned}
(\breve{G}[k] \breve{w})(\tau) & =\int_{0}^{h} g(k h+\tau, \sigma) \breve{w}(\sigma) \mathrm{d} \sigma \\
& =\int_{0}^{h} g(\tau, \sigma-k h) \breve{w}(\sigma) \mathrm{d} \sigma, \quad \tau \in[0, h) .
\end{aligned}
$$

Mapping (10) is a standard discrete-time convolution, describing a shift-invariant system $\breve{\mathcal{G}}$. We call this $\breve{\mathcal{G}}$ the lifting of $\mathcal{G}$.

Example 3.1: Consider the sample-and-hold circuit (Fig. 4), which is the cascade of the ideal sampler and the zero-order hold. This system determines the relation $u(t)=y(\lfloor t / h\rfloor)$, which is clearly not time invariant. Lifting $y$ and $u$ transforms the sample-and-hold circuit into a discrete system, $\breve{u}[k](\tau)=$ $\breve{y}[k](0)$, that is, the $k$ th element of the lifted output is a function of the $k$ th lifted input element only: the impulse response system at $i=0$ acts as $(\breve{G}[0] \breve{y})(\tau)=\breve{y}(0)$ and the others are zero, $\breve{G}[i]=0$. In the lifted domain it is, therefore, a static LTI system.

Although it appears natural to begin with integral representations (6) (because it allows to make the lifting operators concrete), the precise integral form (11) only blurs the reasoning once the advantages of lifting sinks in. One would, therefore, prefer to think of lifted systems purely in discrete time (10) and suppress the finite-horizon time dependence.

Example 3.2: In the same vein, the sample-and-hold circuit from Example 3.1 in the lifted domain may be depicted as in Fig. 5. Here $\mathcal{S}_{\text {Idl }}$ is the lifted ideal sampler transforming a sequence of functions $\{\breve{y}[k]\}$ into a sequence of numbers $\{\bar{y}[k]\}$ as $\bar{y}[k]=\breve{y}[k](0)$ and $\mathcal{H}_{\mathrm{ZOH}}$ is the lifted zero-order hold transforming a sequence of numbers $\{\bar{u}[k]\}$ into a sequence of functions $\{\breve{u}[k]\}$ as $\breve{u}[k](\tau)=\bar{u}[k]$ for all $\tau \in[0, h)$. Both these blocks are static discrete-time LTI systems.

The reasonings of Example 3.2 apply in the general case where each time we leave the discrete signals to what they are and we lift the continuous-time signals to discrete ones. Lifting the input $y$ of the A/D converter $\bar{y}=\mathcal{S} y$ in (3) results in the lifted sampler

$$
\begin{aligned}
\bar{y}=\dot{\mathcal{S}} \breve{y}: \quad \bar{y}[k] & =\sum_{i \in \mathbb{Z}} \int_{0}^{h} \psi((k-i) h-\sigma) \breve{y}[i](\sigma) \mathrm{d} \sigma \\
& =: \sum_{i \in \mathbb{Z}} \dot{S}[k-i] \breve{y}[i]
\end{aligned}
$$

This describes a pure discrete-time shift-invariant system and we think of the operator $S[k]:\left\{[0, h) \rightarrow \mathbb{C}^{n_{y}}\right\} \rightarrow \mathbb{C}^{n_{\bar{y}}}$ as its impulse response. Similarly, the action of the hold device $u=\mathcal{H} \bar{u}$ in (4) after lifting its output becomes

$$
\breve{u}=\grave{\mathcal{H}} \bar{u}: \quad \breve{u}[k]=\sum_{i \in \mathbb{Z}} \grave{H}[k-i] \bar{u}[i]
$$




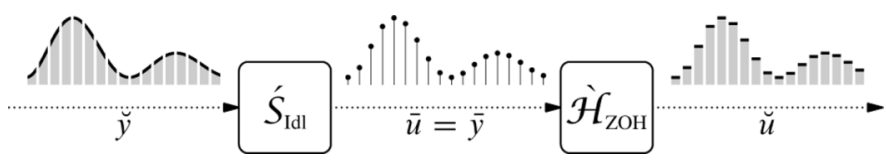

Fig. 5. Sample-and-hold circuit in the lifted domain.

where the operator $\grave{H}[k]: \mathbb{C}^{n_{\bar{u}}} \rightarrow\left\{[0, h) \rightarrow \mathbb{C}^{n_{u}}\right\}$ for each $k$ is a multiplication by the lifted hold function $\breve{\phi}[k]$, i.e., $(\grave{H}[k] \eta)(\tau)=\breve{\phi}[k](\tau) \eta$ for every $\eta \in \mathbb{C}^{n_{\bar{u}}}$. This is also a pure discrete shift-invariant system.

Example 3.3: Consider the predictive first-order hold discussed in Section II-B-II. It has the hold function

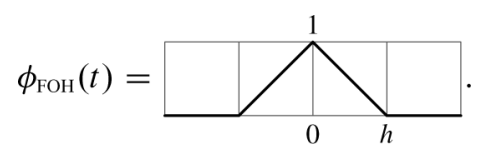

Then the lifted hold $\breve{u}=\grave{\mathcal{H}}_{\mathrm{FOH}} \bar{u}$ is a discrete FIR system with support in $\{-1,0\}$. It maps numbers $\bar{u}[k]$ to functions on $[0, h)$ as follows:

$$
\begin{aligned}
\breve{u}[k] & =\breve{\phi}_{\mathrm{FOH}}[0] \bar{u}[k]+\breve{\phi}_{\mathrm{FOH}}[-1] \bar{u}[k+1] \\
& ={ }_{0}^{\bar{u}[k]} \searrow_{h}+\bigsqcup_{h}^{\bar{u}[k+1]}={ }^{\bar{u}[k]} \square_{0}^{\bar{u}[k+1]},
\end{aligned}
$$

so $\breve{u}[k](\tau)$ is the straight line interpolating $\bar{u}[k]$ and $\bar{u}[k+1]$ at $\tau=0$ and $\tau=h$, respectively.

Remark 3.1: The various lifted systems (operators) that we have seen so far come with different accents to emphasize the dimensionality of their domain and range. The breve accent, such as in $\breve{\mathcal{G}}$, indicates that input and output space at each discrete time is infinite dimensional, $\left\{[0, h) \rightarrow \mathbb{C}^{n}\right\}$. Samplers $\mathcal{S}$ map infinite-dimensional space $\left\{[0, h) \rightarrow \mathbb{C}^{n}\right\}$ to finite-dimensional space $\mathbb{C}^{n}$, which is what the acute accent indicates, and holds $\hat{\mathcal{H}}$ map finite-dimensional space to infinite-dimensional space, indicated by the grave accent. The lifted hybrid signal processor then is a mapping $\breve{\mathcal{F}}_{\mathrm{HSP}}=\grave{\mathcal{H}} \mathcal{\mathcal { S }}$ that goes from an infinite-dimensional space to a finite-dimensional one and back to another infinite-dimensional space again. The accents help in keeping track of the signal space dimensions. When an expression equally applies to either of these types of operators (e.g., in some definitions), we use the tilde, $\tilde{\mathcal{G}}$.

Thus, by lifting all analog signals in the SR setup in Fig. 2 we end up with an equivalent discrete-time setup depicted in Fig. 6. It has two key advantages over the original representation. First, lifting puts continuous- and discrete-time signals on an equal footing. The only difference between "bar" and "breve" discrete signals is that the former are vector (or scalar) valued, whereas the latter are function valued. Conceptually, however, this difference is not more intricate than the difference between scalar and vector signals. Consequently, all systems in Fig. 2, irrespective of whether they are continuous time, discrete time, or hybrid, become pure discrete-time systems. Second, all these

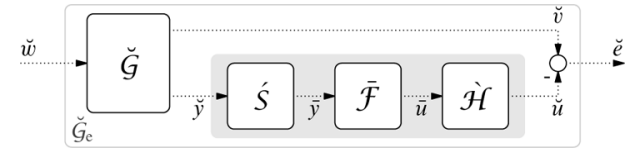

Fig. 6. SR setup in the lifted domain.

discrete systems are now shift invariant, so that many of the familiar LTI notions can be reused almost verbatim.

The advantages come at a cost: the infinite dimensionality of certain input and output signal spaces. Yet this difficulty turns out not to be crucial and can be alleviated by exploiting the structure of the resulting operator-valued mappings.

\section{LIFTING IN FREQUENCY DOMAIN}

With the regained time invariance, we can apply frequency domain methods to lifted $h$-shift-invariant systems and signals.

\section{A. $z$ - and Fourier Transforms}

Naturally, the $z$ - and Fourier transforms of a lifted signal $\breve{f}$ are defined with respect to the discrete time index.

Definition 4.1: The (lifted) z-transform $\mathfrak{Z}\{\breve{f}\}$ of a lifted signal $\breve{f}$ is defined as

$$
\mathfrak{Z}\{\breve{f}\}=\breve{f}(z):=\sum_{k \in \mathbb{Z}} \breve{f}[k] z^{-k}
$$

for all $z \in \mathbb{C}$ for which the series converges.

Definition 4.2: The (lifted) Fourier transform $\mathfrak{F}\{\breve{f}\}$ of a lifted $\breve{f}$ is defined as

$$
\mathfrak{F}\{\breve{f}\}=\breve{f}\left(\mathrm{e}^{\mathrm{j} \theta}\right):=\sum_{k \in \mathbb{Z}} \breve{f}[k] \mathrm{e}^{-\mathrm{j} \theta k},
$$

where $\theta \in[-\pi, \pi]$ is the frequency.

Note that for each $z \in \mathbb{C}$ and $\theta \in[-\pi, \pi]$ the $z$ - and Fourier transforms (if they exist) are still functions of intersample time $\tau \in[0, h)$. This is reflected by the notation $\breve{f}(z ; \tau)$ and $\breve{f}\left(\mathrm{e}^{\mathrm{j} \theta} ; \tau\right)$, which shall be used when these dependences are important. The lifted $z$-transform equals the modified or advanced $z$-transform as introduced by [18], but the intent is entirely different. Also, the lifted Fourier transform is effectively the Zak transform [19] of $f(t)$ modulo scaling.

The following result, which to the best of our knowledge has not explicitly appeared in the literature yet, plays a key role in the subsequent analysis. It is a version of the Poisson Summation Formula, but then one that looses no information about the analog signal. Indeed the point of lifting is to maintain intersample behavior, also in frequency domain.

Theorem 4.1 (Key Lifting Formula): Let $f$ be an analog signal such that $f(t) \mathrm{e}^{-s_{0} t} \in L^{2}(\mathbb{R})$ for some $s_{0} \in \mathbb{C}$. Then

$$
\breve{f}(z ; \tau)=\frac{1}{h} \sum_{k \in \mathbb{Z}} F\left(s_{k}\right) \mathrm{e}^{s_{k} \tau}
$$

for all $\tau \in[0, h)$, where $z:=\mathrm{e}^{s_{0} h}$ and $s_{k}:=s_{0}+\mathrm{j} 2 \omega_{N} k$. 
Proof: Suppose first that $f(t) \mathrm{e}^{-s_{0} t} \in L^{2} \cap L^{1}$. The (regular bilateral) Laplace transform of $f$ is

$$
\begin{aligned}
F(s) & =\int_{-\infty}^{\infty} f(t) \mathrm{e}^{-s t} \mathrm{~d} t=\sum_{k \in \mathbb{Z}} \int_{0}^{h} f(\tau+k h) \mathrm{e}^{-s(\tau+k h)} \mathrm{d} \tau \\
& =\int_{0}^{h} \sum_{k \in \mathbb{Z}} \breve{f}[k](\tau)\left(\mathrm{e}^{s h}\right)^{-k} \mathrm{e}^{-s \tau} \mathrm{d} \tau \\
& =\int_{0}^{h} \breve{f}\left(\mathrm{e}^{s h} ; \tau\right) \mathrm{e}^{-s \tau} \mathrm{d} \tau .
\end{aligned}
$$

Equality (15) now follows by noting that

$$
\frac{1}{h} F\left(s_{k}\right)=\frac{1}{h} \int_{0}^{h}\left[\breve{f}\left(\mathrm{e}^{s_{k} h} ; \tau\right) \mathrm{e}^{-s_{0} \tau}\right] \mathrm{e}^{-\mathrm{j} 2 \omega_{\mathrm{N}} k \tau} \mathrm{d} \tau
$$

is the $k$ th Fourier series coefficient of $\breve{f}\left(\mathrm{e}^{s_{i} h} ; \tau\right) \mathrm{e}^{-s_{0} \tau}$ (mind that $\mathrm{e}^{s_{k} h}=\mathrm{e}^{s_{0} h}=: z$ ).

By Plancherel's theorem [20, Thm.9.13], the assumption that $f(t) \mathrm{e}^{-s_{0} t} \in L^{2}(\mathbb{R})$ assures that (15) holds in $L^{2}$-sense and, therefore, holds pointwise almost everywhere.

Remark 4.1: Equality (16) might bear a resemblance to [12, Eq. (11.5.1)], with (14) playing the role of [12, Eq. (11.5.2)]. This suggests that the lifting can be thought of as an analog counterpart of the polyphase representation (known actually as the discrete lifting in the control literature [7]). Thus, $\breve{f}\left(\mathrm{e}^{s h}, \tau\right)$ can be viewed as the $\tau$ th polyphase component of $F(s)$. This analogy is useful in comprehending properties of systems in the lifted domain discussed below.

A particular case of the key lifting formula for imaginary $s_{0}=j \theta / h$ says that there is a bijection from the lifted Fourier transform $\breve{f}\left(\mathrm{e}^{\mathrm{j} \theta}\right)$ and the classical Fourier transform $F(\mathrm{j} \omega)$ :

$$
\begin{aligned}
F\left(\mathrm{j} \omega_{k}\right) & =\int_{0}^{h} \breve{f}\left(\mathrm{e}^{\mathrm{j} \theta} ; \tau\right) \mathrm{e}^{-\mathrm{j} \omega_{k} \tau} \mathrm{d} \tau, \\
\breve{f}\left(\mathrm{e}^{\mathrm{j} \theta} ; \tau\right) & =\frac{1}{h} \sum_{k \in \mathbb{Z}} F\left(\mathrm{j} \omega_{k}\right) \mathrm{e}^{\mathrm{j} \omega_{k} \tau},
\end{aligned}
$$

for any square integrable $f$, where

$$
\omega_{k}:=\frac{\theta+2 \pi k}{h}=\frac{\theta}{h}+2 \omega_{N} k
$$

are aliased frequencies.

Remark 4.2: A special case of (17b) corresponding to $\tau=0$ yields the classical formula connecting Fourier transforms of an analog signal (provided it is continuous and satisfies some other mild conditions [21]) and its sampled version: $\mathfrak{F}\{\bar{f}\}=$ $(1 / h) \sum_{i \in \mathbb{Z}} F\left(j \omega_{i}\right)$. We believe that the derivation via the use of lifting and (17b) is somewhat cleaner and more intuitive than the conventional impulse-train modulation [22] or "reverse engineering" [23] arguments.

Equations (17) are very useful when lifted Fourier transforms need to be determined or its inverse, and as we shall see in [17, Sec. V] it is a key technical tool in the design of optimal samplers and holds.

Example 4.1: To illustrate a use of (17b), let $f(t)=$ $(1 / h) \operatorname{sinc}_{h}(t)$. Since $F(j \omega)=\mathbb{1}_{\left[-\omega_{N}, \omega_{N}\right]}(\omega)$, equality $(17 \mathrm{~b})$ yields the lifted Fourier transform $\breve{f}\left(\mathrm{e}^{\mathrm{i} \theta} ; \tau\right)=(1 / h) \mathrm{e}^{\mathrm{j} \theta \tau / h}$ for $\theta \in[-\pi, \pi]$ and $\tau \in[0, h)$.

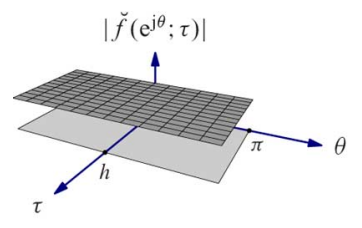

(a)

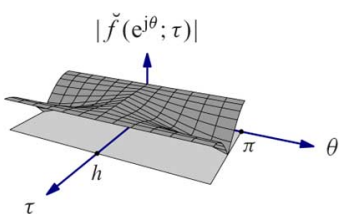

(b)
Fig. 7. Amplitude $\left|\breve{f}\left(\mathrm{e}^{\mathrm{j} \theta} ; \tau\right)\right|$ versus $\theta$ and $\tau$. (a) $f(t)=\operatorname{sinc}_{h}(t)$. (b) $f(t)=$ $\operatorname{sinc}_{h}^{2}(t)$.

Example 4.2: The Fourier transform of $f(t)=$ $(1 / h) \operatorname{sinc}_{h}^{2}(t)$ is the "tent"

$$
F(j \omega)=\left(1-|\omega| /\left(2 \omega_{N}\right)\right) \mathbb{1}_{\left[-2 \omega_{N}, 2 \omega_{N}\right]}(\omega) .
$$

Then

$$
\begin{gathered}
\breve{f}\left(\mathrm{e}^{\mathrm{j} \theta} ; \tau\right)= \\
(1 / h) \mathrm{e}^{j \theta \tau / h}\left(1-(|\theta| / 2 \pi)+(|\theta| / 2 \pi) \mathrm{e}^{-\mathrm{j} 2 \omega_{N} \tau \operatorname{sign} \theta}\right)
\end{gathered}
$$

for $\theta \in[-\pi, \pi]$ and $\tau \in[0, h)$.

Fig. 7 depicts the amplitude $\left|f\left(\mathrm{e}^{\mathrm{j} \theta} ; \tau\right)\right|$ as a function of $\theta \in$ $[-\pi, \pi]$ and $\tau \in[0, h)$ for the functions considered in the above two examples. Such amplitude plots demonstrate how the amplitude spectrum of the sampled signal $f(k h+\tau)$ changes with time offset $\tau$ (for the $\operatorname{sinc}_{h}$ it does not change).

\section{B. Transfer Function and Frequency Response}

It is well known that convolution (dynamic) systems become algebraic (static) if considered in the transform domain. This is also true for lifted systems as we shall see with the introduction of the lifted transfer function formalism.

The transfer function $\breve{G}(z)$ of the lifted system (10) is formally defined as the $z$-transform of its impulse response

$$
\breve{G}(z):=\sum_{i \in \mathbb{Z}} \breve{G}[i] z^{-i} .
$$

A standard index change in (10) then shows [24] that the lifted $z$-transforms of input and output satisfy the familiar

$$
\breve{u}(z)=\breve{G}(z) \breve{y}(z) .
$$

It is worth recalling that the lifted impulse response $\breve{G}[k]$ for each $k \in \mathbb{Z}$ is an integral operator of the form (11). Hence, so is the lifted transfer function $\breve{G}(z)$. It can be shown that the "multiplication" in (20) should be understood as

$$
\breve{u}(z ; \tau)=\int_{0}^{h} \breve{g}(z ; \tau, \sigma) \breve{y}(z ; \sigma) \mathrm{d} \sigma, \quad \tau \in[0, h)
$$

where $\breve{g}(z ; \tau, \sigma)$ is the lifted $z$-transform of the impulse response kernel $g(t, s)$ of $\mathcal{G}$ with respect to its first variable ${ }^{3}$,

$$
\breve{g}(z ; \tau, \sigma):=\sum_{k \in \mathbb{Z}} g(\tau+k h, \sigma) z^{-k} .
$$

Again we want to make the point here that (20) is more in the spirit of lifting than the gritty details of (21) and (22).

\footnotetext{
${ }^{3}$ Alternatively, the " $1 / z$-transform" with respect to its second variable.
} 
Example 4.3: In Example 3.2 we showed that the impulse response $\breve{G}[k]$ of the cascade of the ideal sampler and the zeroorder hold is such that $(\breve{G}[0] \breve{y})(\tau)=\breve{y}(0)$ and with all other $\breve{G}[k]$ zero. Therefore, the transfer function of this cascade in the lifted domain acts as $\breve{G}(z) \breve{y}(z)=\breve{y}(z ; 0)$.

"Semilifted" elements, such as lifted sampler and hold, can be described in terms of their lifted transfer functions in the same way. The only difference from the case considered above is that either output or input space is now finite dimensional. Thus, the transfer function $S^{\prime}(z)$ of the lifted sampler $\mathcal{S}$ in (12) is a linear functional from $\left\{[0, h) \rightarrow \mathbb{C}^{n_{y}}\right\}$ to $\mathbb{C}^{n_{\bar{y}}}$ of the form ${ }^{4}$

$$
\bar{y}(z)=\dot{S}(z) \breve{y}(z): \quad \bar{y}(z)=\int_{0}^{h} \breve{\psi}(z ;-\sigma) \breve{y}(z ; \sigma) \mathrm{d} \sigma
$$

for each $z \in \mathbb{C}$ where it is defined. Here $\breve{\psi}(z)$ is the lifted $z$-transform of the sampling function $\psi(t)$. Similarly, the transfer function $\grave{H}(z)$ of the lifted hold $\dot{\mathcal{H}}$ in (13) is an operator from $\mathbb{C}^{n_{\bar{u}}}$ to $\left\{[0, h) \rightarrow \mathbb{C}^{n_{u}}\right\}$ of the form

$$
\breve{u}(z)=\grave{H}(z) \bar{u}(z): \quad \breve{u}(z ; \tau)=\breve{\phi}(z ; \tau) \bar{u}(z)
$$

for each $z \in \mathbb{C}$ where it is defined. Here, $\breve{\phi}(z)$ is the lifted $z$-transform of the hold function $\phi(t)$.

Example 4.4: Consider again the predictive first-order hold $\mathcal{H}_{\mathrm{FOH}}$ studied in Example 3.3. Inspecting the formulas in this example, it is readily seen that

$$
\breve{\phi}_{\mathrm{FOH}}(z ; \cdot)=\breve{\phi}_{\mathrm{FOH}}[-1] z+\breve{\phi}_{\mathrm{FOH}}[0]=\sum_{0} z+\coprod_{0} \cdot
$$

The "static gain" of this transfer function is $\grave{H}_{\mathrm{FOH}}(1 ; \tau) \equiv 1$, which agrees with our understanding of this hold.

Obviously, $\breve{G}\left(\mathrm{e}^{\mathrm{j} \theta}\right)$ will be referred to as the (lifted) frequency response and the transfer kernel $\breve{g}\left(\mathrm{e}^{\mathrm{j} \theta}\right)$ as its frequency response kernel. It maintains the familiar interpretation in the sense that for any fixed $\theta \in[-\pi, \pi]$ the response $\breve{u}=\breve{\mathcal{G}} \breve{y}$ to a (lifted) harmonic function $\breve{y}[k]=\mathrm{e}^{\mathrm{j} \theta k} \breve{w}$ (with $\breve{w}:[0, h) \rightarrow \mathbb{C}^{n_{w}}$ ) if it exists, is again harmonic [25], $\breve{u}[k]=\mathrm{e}^{\mathrm{j} \theta k} G\left(\mathrm{e}^{\mathrm{j} \theta}\right) \breve{w}$. The absolute value $|\breve{y}[k](\tau)|$ of a harmonic input does not depend on $k$ and neither does the output. As shown in [25], if the magnitude of harmonic $\breve{y}[k]$ (for whatever $k$ ) is measured in $L^{2}[0, h$ )-sense then the maximal possible magnitude gain (power gain) at frequency $\theta$ equals the largest singular value of $G\left(\mathrm{e}^{\mathrm{j} \theta}\right)$ as defined later on in this paper, (30). This is very similar to the interpretation of the conventional frequency response of discrete-time systems.

Example 4.5: Consider the sinc-sampler $\mathcal{S}_{\text {sinc }}$ (see Section II-B-I) having the sampling function $\psi_{\text {sinc }}(t)=$ $(1 / h) \operatorname{sinc}_{h}(t)$. Example 4.1 then yields that the frequency response kernel of $S_{\text {sinc }}\left(\mathrm{e}^{\mathrm{j} \theta}\right)$ is $\breve{\psi}_{\text {sinc }}\left(\mathrm{e}^{\mathrm{j} \theta} ;-\sigma\right)=(1 / h) \mathrm{e}^{-\mathrm{j} \theta \sigma / h}$. $\nabla$

Example 4.6: The hold function of the sinc-hold $\mathcal{H}_{\text {sinc }}$ (see Section II-B-II) is $\phi_{\text {sinc }}(t)=\operatorname{sinc}_{h}(t)$. Therefore, the frequency response kernel of $\grave{H}_{\text {sinc }}\left(e^{j \theta}\right)$ is $\breve{\phi}_{\text {sinc }}\left(\mathrm{e}^{\mathrm{j} \theta} ; \tau\right)=\mathrm{e}^{\mathrm{j} \theta \tau / h}$.

\footnotetext{
${ }^{4}$ Strictly speaking, it should be $z^{-1} \breve{\psi}(z ; h-\sigma)$, rather than $\breve{\psi}(z ;-\sigma)$ (these two are equivalent), because the intersample time variable lies in $[0, h]$. We, however, prefer to trade notational rigor for simplicity in this case.
}

\section{SPACES AND NORMS}

This section reviews the notions of signal and system norms in the lifted domain. Most results presented below are either known or quite straightforward extensions of known results that can be found in, e.g., [7], [8, Ch. 2], [14, Appendix A].

\section{A. Signal Spaces and Norms}

As the lifting transformation is merely a different viewpoint of analog signals, we can take it to be norm preserving. Concretely, the $L^{2}$ signal norm translates to the lifted domain as follows:

$$
\begin{aligned}
\|f\|_{2}^{2} & =\int_{-\infty}^{\infty}|f(t)|^{2} \mathrm{~d} t=\sum_{k \in \mathbb{Z}} \int_{0}^{h}|\breve{f}[k](\tau)|^{2} \mathrm{~d} \tau \\
& =\sum_{k \in \mathbb{Z}}\|\breve{f}[k]\|_{\mathbb{L}}^{2}=:\|\breve{f}\|_{2}^{2}
\end{aligned}
$$

where $\mathbb{L}:=L^{2}[0, h)$. By analogy with the standard $\ell^{2} \mathbb{C}(\mathbb{Z})$ space, we call the quantity defined by (25) the $\ell^{2}$-norm of $f$ (this is a norm, just because so is the $L^{2}$-norm in continuous time) and denote the set of all lifted signals having a bounded $\ell^{2}$-norm as $\ell_{\mathbb{L}}^{2}(\mathbb{Z})$, which is a Hilbert space with the obvious inner product. Thus lifting by construction is an isometric isomorphism between $L^{2} \mathbb{C}(\mathbb{R})$ and $\ell^{2} \mathbb{L}(\mathbb{Z})$.

Remark 5.1: All signals in the lifted SR scheme in Fig. 6 are now measured by various $\ell^{2}$-norms. The only difference between these norms is in their "subscript spaces": $\mathbb{C}$ or $\mathbb{L}$. This difference, however, is peripheral, so we hereafter drop the subscript from the notation for $\ell^{2}$ and related spaces.

$\nabla$

With a slight abuse of notation we use $\ell^{2}\left(\mathbb{Z}_{l}^{+}\right)$and $\ell^{2}\left(\mathbb{Z}_{l}^{-}\right)$to denote the subspaces of $\ell^{2}(\mathbb{Z})$ consisting of signals that are zero in $\mathbb{Z}_{l}^{-}$and $\mathbb{Z}_{l}^{+}$, respectively. Clearly, $\ell^{2}(\mathbb{Z})=\ell^{2}\left(\mathbb{Z}_{l}^{+}\right) \oplus \ell^{2}\left(\mathbb{Z}_{l}^{-}\right)$ for every integer $l$. We shall need these subspaces later on to discuss causality.

We also need corresponding frequency-domain spaces. Let $\mathbb{K}$ stand for either $\mathbb{C}^{n}$ or $\mathbb{L}$, depending on whether our signal is a plain discrete-time signal or a lifted one. The Hilbert space $L^{2}(\mathbb{T})$ is the set of functions $\tilde{f}(z): \mathbb{T} \rightarrow \mathbb{K}$, for which ${ }^{5}$

$$
\|\tilde{f}\|_{2}:=\left(\frac{1}{2 \pi} \int_{-\pi}^{\pi}\left\|\tilde{f}\left(\mathrm{e}^{\mathrm{j} \theta}\right)\right\|_{\mathbb{K}}^{2} \mathrm{~d} \theta\right)^{1 / 2}<\infty .
$$

The Hardy space $H^{2}$ is the set of functions $\tilde{f}(z): \mathbb{C} \backslash \overline{\mathbb{D}} \rightarrow \mathbb{K}$ which are analytic and satisfy

$$
\|\tilde{f}\|_{H^{2}}:=\sup _{\rho>1}\left(\frac{1}{2 \pi} \int_{-\pi}^{\pi}\left\|\tilde{f}\left(\rho \mathrm{e}^{\mathrm{j} \theta}\right)\right\|_{\mathbb{K}}^{2} \mathrm{~d} \theta\right)^{1 / 2}<\infty .
$$

The domain of functions in $H^{2}$ can be extended to $\mathbb{T}$ and the result is a closed subspace of $L^{2}(\mathbb{T})$ with $\|\tilde{f}\|_{H^{2}}=\|\tilde{f}\|_{2}$. The orthogonal complement of $H^{2}$ in $L^{2}(\mathbb{T})$ is denoted by $H_{\perp}^{2}$ and is comprised of analytic and bounded functions $\tilde{f}(z): \mathbb{D} \rightarrow \mathbb{K}$ such that $\|\tilde{f}\|_{2}<\infty$. Finally, by $z^{l} H^{2}$ we denote the space of functions $\tilde{f}(z): \mathbb{C} \backslash \overline{\mathbb{D}} \rightarrow \mathbb{K}$ such that $z^{-l} \breve{f}(z) \in H^{2}$.

\footnotetext{
${ }^{5}$ We use the same norm symbol for several time- and frequency-domain norms. Context determines which is intended.
} 
The Parseval's identity, which is instrumental in converting energy-based optimization problems to the frequency domain, also extends to general $\ell^{2}$ spaces. Namely, for any $\tilde{f} \in \ell^{2}(\mathbb{Z})$ we have that $\mathfrak{F}\{\tilde{f}\} \in L^{2}(\mathbb{T})$ and

$$
\|\tilde{f}\|_{2}=\|\mathfrak{F}\{\tilde{f}\}\|_{2}
$$

The Fourier transform is thus an isometric isomorphism between $\ell^{2}(\mathbb{Z})$ and $L^{2}(\mathbb{T})$. Similarly the $z$-transform is an isometric isomorphism between $\ell^{2}\left(\mathbb{Z}_{l}^{+}\right)$and $z^{l} H^{2}$ for any $l$.

Example 5.1: Consider $f(t)=(1 / h) \operatorname{sinc}_{h}(t)$. By Example 4.1, $\|f\|_{2}$ can also be computed via the $L^{2}(\mathbb{T})$-norm of its lifted Fourier transform:

$$
\|\breve{f}\|_{2}^{2}=\frac{1}{2 \pi} \int_{-\pi}^{\pi}\left\|\frac{1}{h} \mathrm{e}^{\mathrm{j} \theta \tau / h}\right\|_{\mathbb{L}}^{2} \mathrm{~d} \theta=\frac{1}{2 \pi} \int_{-\pi}^{\pi} \frac{1}{h} \mathrm{~d} \theta=\frac{1}{h},
$$

which agrees with the direct computation of $\|f\|_{2}^{2}$.

\section{B. Adjoint Systems and Conjugate Transfer Functions}

Since both lifting and Fourier transformation preserve inner products, the adjoint of an operator is equivalent in all domains, i.e., the lifting of the adjoint operator is the adjoint of the lifted operator, and likewise for the Fourier transformed operator. It is well known that the kernel of the adjoint of $\mathcal{G}$, given in (6), is

$$
g^{\sim}(t, s):=[g(s, t)]^{*}
$$

with ${ }^{*}$ here denoting complex conjugate transpose. The conjugate operator $\sim$ defined by (26) not only takes the complex conjugate transpose of the matrix but also interchanges the two time parameters. It is more generally defined for frequency depending functions as

$$
\breve{g}^{\sim}(z ; \tau, \sigma):=\left[\breve{g}\left(\frac{1}{\bar{z}} ; \sigma, \tau\right)\right]^{*}
$$

for then the $z$-transform of the conjugate is the conjugate of the $z$-transform (with respect to the first variable)

$$
\begin{aligned}
\mathfrak{Z}\left\{g^{\sim}(\tau, \sigma)\right\} & =\sum_{k \in \mathbb{Z}} g^{\sim}(\tau+k h, \sigma) z^{-k} \\
& =\sum_{m=-k}\left[g(\sigma, \tau-m h) \bar{z}^{m}\right]^{*} \\
& =\sum_{m \in \mathbb{Z}}\left[g(\sigma+m h, \tau)\left(\frac{1}{\bar{z}}\right)^{-m}\right]^{*}=\breve{g}^{\sim}(z ; \tau, \sigma) .
\end{aligned}
$$

According to (21), (22), and the above, $\breve{g}^{\sim}(z ; \tau, \sigma)$, hence, is the kernel of the transfer function of the adjoint system $\mathcal{G}^{*}$. We denote this transfer function as $\breve{G}^{\sim}(z)$

$$
\breve{y}(z)=\breve{G}^{\sim}(z) \breve{u}(z): \quad \breve{y}(z ; \tau)=\int_{0}^{h} \breve{g} \sim(z ; \tau, \sigma) \breve{u}(z ; \sigma) d \sigma .
$$

It is readily seen that for $z=\mathrm{e}^{\mathrm{j} \theta}$ the conjugate $\breve{G}^{\sim}\left(\mathrm{e}^{\mathrm{j} \theta}\right)$ is the adjoint of $\breve{G}\left(\mathrm{e}^{\mathrm{j} \theta}\right)$ with respect to $\mathbb{L}$

$$
\left\langle\breve{u}\left(\mathrm{e}^{\mathrm{j} \theta}\right), \breve{G}\left(\mathrm{e}^{\mathrm{j} \theta}\right) \breve{y}\left(\mathrm{e}^{\mathrm{j} \theta}\right)\right\rangle_{\mathbb{L}}=\left\langle\breve{G}^{\sim}\left(\mathrm{e}^{\mathrm{j} \theta}\right) \breve{u}\left(\mathrm{e}^{\mathrm{j} \theta}\right), \breve{y}\left(\mathrm{e}^{\mathrm{j} \theta}\right)\right\rangle_{\mathbb{L}} .
$$

That is, the lifted transfer function of the adjoint equals the adjoint of the lifted transfer function.

Now, the adjoint of the sampler in (3) can be derived via

$$
\begin{aligned}
\langle\mathcal{S} y, \bar{u}\rangle_{\ell^{2}} & =\sum_{i \in \mathbb{Z}} \bar{u}^{*}[i] \int_{-\infty}^{\infty} \psi(i h-s) y(s) \mathrm{d} s \\
& =\int_{-\infty}^{\infty}\left(\sum_{i \in \mathbb{Z}}[\psi(i h-s)]^{*} \bar{u}[i]\right)^{*} y(s) \mathrm{d} s \\
& =\left\langle y, \mathcal{S}^{*} \bar{u}\right\rangle_{L^{2}} .
\end{aligned}
$$

Thus, the adjoint of $\mathcal{S}$ with a sampling function $\psi(t)$ is a $\mathcal{H}$ with the hold function $\phi(t)=[\psi(-t)]^{*}=: \psi^{\sim}(t)$ [the latter is just an LTI version of (26)]. This prompts a duality between the A/D and D/A conversions and also implies that the adjoint of $\mathcal{H}$ with $\phi(t)$ is $\mathcal{S}$ with $\psi(t)=\phi^{\sim}(t)$. The conjugate transfer function of $\dot{S}(z), S^{\sim}(z)$, is the following lifted hold:

$$
\breve{y}(z)=\dot{S}^{\sim}(z) \bar{u}(z): \quad \breve{y}(z ; \tau)=\breve{\psi} \sim(z ; \tau) \bar{u}(z),
$$

with $\breve{\psi}^{\sim}(z ; \tau):=[\breve{\psi}(1 / \bar{z} ;-\tau)]^{*}$. The conjugate transfer function of $\dot{H}(z)$ is

$$
\bar{y}(z)=\grave{H}^{\sim}(z) \breve{u}(z): \quad \bar{y}(z)=\int_{0}^{h} \breve{\phi} \sim(z ;-\sigma) \breve{u}(z) \sigma d \sigma,
$$

which is a lifted sampler.

The following result will be used in the next part.

Proposition 5.1: Let $\mathcal{S}$ be a sampler, the sampling function $\psi(t)$ of which is such that $\psi(t) \mathrm{e}^{-\sigma t} \in L^{2}(\mathbb{R})$ for some $\sigma \in \mathbb{R}$. Then whenever $|z|=\mathrm{e}^{\sigma h}$

$$
\begin{aligned}
\dot{S}(z) \dot{S}^{\sim}(z) & =\int_{0}^{h} \breve{\psi}(z ; \tau)\left[\breve{\psi}\left(\frac{1}{\bar{z}} ; \tau\right)\right]^{*} \mathrm{~d} \tau \\
& =\frac{1}{h} \sum_{k \in \mathbb{Z}} \Psi\left(s_{k}\right) \Psi^{\sim}\left(s_{k}\right),
\end{aligned}
$$

where $\Psi(s)$ is the bilateral Laplace transform of $\psi$ and $s_{k}$ are as defined in Theorem 4.1.

Proof: Equality (27a) follows by routine substitution. To derive (27b), denote the integral in (27a) by $M$ and use (17b)

$$
\begin{aligned}
M & =\frac{1}{h^{2}} \int_{0}^{h} \sum_{k \in \mathbb{Z}} \Psi\left(s_{k}\right) \mathrm{e}^{s_{k} \tau}\left[\sum_{i \in \mathbb{Z}} \Psi\left(s_{i}\right) \mathrm{e}^{s_{i} \tau}\right]^{\sim} \mathrm{d} \tau \\
& =\frac{1}{h^{2}} \sum_{k \in \mathbb{Z}} \Psi\left(s_{k}\right) \sum_{i \in \mathbb{Z}} \Psi^{\sim}\left(s_{i}\right) \int_{0}^{h} \mathrm{e}^{\mathrm{j} 2 \omega_{N}(k-i) \tau} \mathrm{d} \tau .
\end{aligned}
$$

The result now follows by $\int_{0}^{h} \mathrm{e}^{\mathrm{j} 2 \omega_{N}(k-i) \tau} d \tau=h \bar{\delta}[k-i]$.

An immediate corollary of this result is that if $\psi(t)$ is scalar, then $S^{\prime}\left(\mathrm{e}^{\mathrm{j} \theta}\right) \dot{S}^{\sim}\left(\mathrm{e}^{\mathrm{j} \theta}\right)=\left\|\breve{\psi}\left(\mathrm{e}^{\mathrm{j} \theta}\right)\right\|_{\mathbb{L}}^{2}=(1 / h) \sum_{k \in \mathbb{Z}}\left|\Psi\left(\mathrm{j} \omega_{k}\right)\right|^{2}$, where $\omega_{k}$ are defined by (18). Also, by duality we have:

Proposition 5.2: Let $\mathcal{H}$ be a hold, the hold function $\phi(t)$ of which is such that $\phi(t) \mathrm{e}^{-\sigma t} \in L^{2}(\mathbb{R})$ for some $\sigma \in \mathbb{R}$. Then 
whenever $|z|=\mathrm{e}^{\sigma h}$

$$
\begin{aligned}
\grave{H}^{\sim}(z) \grave{H}(z) & =\int_{0}^{h}\left[\breve{\phi}\left(\frac{1}{\bar{z}} ; \tau\right)\right]^{*} \breve{\phi}(z ; \tau) \mathrm{d} \tau \\
& =\frac{1}{h} \sum_{k \in \mathbb{Z}} \Phi^{\sim}\left(s_{k}\right) \Phi\left(s_{k}\right)
\end{aligned}
$$

where $\Phi(s)$ is the bilateral Laplace transform of $\phi$.

\section{C. $L^{\infty}$ System Norm}

The $L^{\infty}$ norm [cf. (2)] of a lifted transfer function $\tilde{G}(z)$ : $\mathbb{K}_{\mathrm{i}} \rightarrow \mathbb{K}_{\mathrm{o}}$ is defined as

$$
\|\tilde{G}\|_{\infty}:=\operatorname{ess}_{\theta \in[-\pi, \pi]} \sup _{\max }\left[\tilde{G}\left(\mathrm{e}^{\mathrm{j} \theta}\right)\right]<\infty
$$

where the (operator) maximal singular value $\sigma_{\max }$ equals

$$
\sigma_{\max }\left[\tilde{G}\left(\mathrm{e}^{\mathrm{j} \theta}\right)\right]=\sup _{\tilde{y} \in \mathbb{K}_{\mathrm{i}},\|\tilde{y}\|_{\mathbb{K}_{\mathrm{i}}}=1}\left\|\tilde{G}\left(\mathrm{e}^{\mathrm{j} \theta}\right) \tilde{y}\right\|_{\mathbb{K}_{\mathrm{o}}}
$$

i.e., (30) is the induced norm of $\tilde{G}\left(\mathrm{e}^{\mathrm{j} \theta}\right)$. If $\tilde{G}(z)$ is the transfer function of an LTI system $\tilde{\mathcal{G}}$, we also refer to (29) as the $L^{\infty}$-norm of the system and denote it as $\|\tilde{\mathcal{G}}\|_{\infty}$. For given $\mathbb{K}_{\mathrm{i}}$ and $\mathbb{K}_{\mathrm{O}}$ the vector space of all transfer functions with finite $L^{\infty}$-norm is represented with the same symbol $L^{\infty}$, so

$$
L^{\infty}=\left\{\tilde{G}: \mathbb{T} \rightarrow\left(\mathbb{K}_{\mathrm{i}} \rightarrow \mathbb{K}_{\mathrm{o}}\right) \mid\|\tilde{G}\|_{\infty}<\infty\right\} .
$$

By the arguments of [24], it can be shown that $\|\breve{\mathcal{G}}\|_{\infty}$ equals the $L^{2}(\mathbb{R})$-induced norm of its original, $\mathcal{G}$, i.e., $\|\breve{\mathcal{G}}\|_{\infty}=\sup _{\|y\|_{2}=1}\|\mathcal{G} y\|_{2}$. Its square, $\|\breve{\mathcal{G}}\|_{\infty}^{2}$, is therefore the maximal energy gain of the system and also equals the maximal power gain. Likewise, $\|\mathcal{S}\|_{\infty}$ and $\|\hat{H}\|_{\infty}$ equal $L^{2}(\mathbb{R}) \rightarrow \ell^{2}(\mathbb{Z})$ and $\ell^{2}(\mathbb{Z}) \rightarrow L^{2}(\mathbb{R})$ induced norms of $\mathcal{S}$ and $\mathcal{H}$, respectively.

Example 5.2: Consider the HSP $\mathcal{H}_{\mathrm{ZOH}} \mathcal{S}_{\epsilon}$, where $\mathcal{S}_{\epsilon}$ is the "almost ideal" sampler with $\psi_{\epsilon}(t)=(1 / \epsilon) \mathbb{1}_{[0, \epsilon]}(t)$ for $0<\epsilon<$ $h$ (the smaller $\epsilon$ is, the more this sampler behaves like the ideal sampler). Because $\psi_{\epsilon}(t)$ is scalar, by Proposition 5.1 (this can also be seen via the Riesz-Fréchet theorem) we have that

$$
\left\|\dot{\mathcal{S}}_{\epsilon}\right\|_{\infty}=\sup _{\theta \in[-\pi, \pi]}\left\|\breve{\psi}_{\epsilon}\left(\mathrm{e}^{\mathrm{j} \theta}\right)\right\|_{\mathbb{L}}=\sup _{\theta \in[-\pi, \pi]}\left\|\frac{1}{\epsilon} \mathbb{1}_{[0, \epsilon]}\right\|_{\mathbb{L}}=\frac{1}{\sqrt{\epsilon}} .
$$

In fact, the maximizing input having the unity norm for this system is $y_{\max }(t)=1 / \sqrt{\epsilon} \mathbb{1}_{[h-\epsilon, h)}(t)$ and is unique (modulo sign and $h$-shifts). Regarding $\mathcal{H}_{\mathrm{ZOH}}$, it is readily seen that $\|u\|_{2}=\sqrt{h}\|\bar{u}\|_{2}$ for every $\bar{u} \in \ell^{2}(\mathbb{Z})$. Thus, $\left\|\grave{\mathcal{H}}_{\mathrm{ZOH}}\right\|_{\infty}=\sqrt{h}$ and any input $\bar{u}$ is maximizing. Hence, $y_{\max }$ actually maximizes the energy gain of the overall HSP $\mathcal{H}_{\mathrm{ZOH}} \mathcal{\mathcal { S }}_{\epsilon}$ and we have

$$
\left\|\grave{\mathcal{H}}_{\mathrm{ZOH}} \dot{\mathcal{S}}_{\epsilon}\right\|_{\infty}=\sqrt{h}\left\|\dot{\mathcal{S}}_{\epsilon}\right\|_{\infty}=\sqrt{\frac{h}{\epsilon}} .
$$

It becomes unbounded as $\epsilon \downarrow 0$, like in the $L^{2}$ case.
Another space we need is the Hardy space $H^{\infty}$. It is defined as the set of transfer functions $\tilde{G}(z)$, which are analytic for $z \in$ $\mathbb{C} \backslash \overline{\mathbb{D}}$ and satisfy

$$
\|\tilde{G}\|_{H^{\infty}}:=\operatorname{ess}_{z \in \mathbb{C} \backslash \overline{\mathbb{D}}} \sigma_{\max }[\tilde{G}(z)]<\infty .
$$

Like in the case with the $H^{2}$ signal space, $H^{\infty}$ operators can be extended to $z \in \mathbb{T}$, resulting in a closed subspace of $L^{\infty}$ with $\|\tilde{G}\|_{H^{\infty}}=\|\tilde{G}\|_{\infty}$. By $z^{l} H^{\infty}$ we then denote the subspace of $L^{\infty}$ consisting of operators $\tilde{G}(z)$ such that $z^{-l} \tilde{G}(z) \in H^{\infty}$. Loosely speaking, $H^{\infty}$ is the space of transfer functions, which are analytic and bounded in $\mathbb{C} \backslash \mathbb{D}$, whereas $z^{l} H^{\infty}$ is the space of analytic transfer functions with relaxed (if $l>0$ ) or tightened (if $l<0$ ) boundedness in $|z| \rightarrow \infty$.

\section{D. $L^{2}$ System Norm}

The $L^{2}$ norm [cf. (1)] of lifted (or semi-lifted) transfer functions $\tilde{G}(z): \mathbb{K}_{\mathrm{i}} \rightarrow \mathbb{K}_{\mathrm{o}}$ is defined as

$$
\|\tilde{G}\|_{2}:=\left(\frac{1}{2 \pi h} \int_{-\pi}^{\pi}\left\|\tilde{G}\left(\mathrm{e}^{\mathrm{j} \theta}\right)\right\|_{\mathrm{HS}}^{2} \mathrm{~d} \theta\right)^{1 / 2}<\infty
$$

(the scaling factor will become clear soon, it is not present in the standard discrete case). Here $\|\cdot\|_{H S}$ is the Hilbert-Schmidt operator norm, which can in general be calculated as

$$
\begin{aligned}
\left\|\tilde{G}\left(\mathrm{e}^{\mathrm{j} \theta}\right)\right\|_{\mathrm{HS}}^{2} & =\operatorname{tr}\left[\tilde{G}\left(\mathrm{e}^{\mathrm{j} \theta}\right) \tilde{G}^{\sim}\left(\mathrm{e}^{\mathrm{j} \theta}\right)\right]=\operatorname{tr}\left[\tilde{G}^{\sim}\left(\mathrm{e}^{\mathrm{j} \theta}\right) \tilde{G}\left(\mathrm{e}^{\mathrm{j} \theta}\right)\right] \\
& =\sum_{i} \sigma_{i}^{2}\left[\tilde{G}\left(\mathrm{e}^{\mathrm{j} \theta}\right)\right]
\end{aligned}
$$

with $\sigma_{i}[\cdot]$ the $i$ th singular value. For integral operators $\mathbb{L} \rightarrow \mathbb{L}$ as in (21) we have that

$$
\left\|\breve{G}\left(\mathrm{e}^{\mathrm{j} \theta}\right)\right\|_{\mathrm{HS}}^{2}=\int_{0}^{h} \int_{0}^{h}\left\|\breve{g}\left(\mathrm{e}^{\mathrm{j} \theta} ; \tau, \sigma\right)\right\|_{F}^{2} \mathrm{~d} \tau \mathrm{d} \sigma .
$$

For semi-lifted operators, like $S(z)$ and $\grave{H}(z)$, the calculations of the Hilbert-Schmidt norm reduce to the computation of the matrix trace (cf. Propositions 5.1 and 5.2). If $\tilde{G}(z)$ is the transfer function a (semi-) lifted system $\tilde{\mathcal{G}}$ we also refer to (31) as the $L^{2}$-norm of the system and denote it as $\|\tilde{\mathcal{G}}\|_{2}$. The vector space of systems with finite $L^{2}$ system norm (31) is represented simply as $L^{2}$

$$
L^{2}=\left\{\tilde{G}: \mathbb{T} \rightarrow\left(\mathbb{K}_{\mathrm{i}} \rightarrow \mathbb{K}_{\mathrm{o}}\right)\|\| \tilde{G} \|_{2}<\infty\right\}
$$

In contrast with the ordinary $L^{2}$ norm for LTI-systems, the $L^{2}$ system norm is not equivalent to a signal norm, even though we use the same notation, $\|\cdot\|_{2}$ and $L^{2}$. Neither of the two system spaces $L^{\infty}$ and $L^{2}$ is a subset of the other. However, if the rank of the transfer function is uniformly bounded then being in $L^{\infty}$ implies being in $L^{2}$.

Proposition 5.3: Let $\tilde{G} \in L^{\infty}$ be such that $\operatorname{rank} \tilde{G}\left(\mathrm{e}^{\mathrm{j} \theta}\right) \leq r$ for almost all $\theta \in[-\pi, \pi]$ and some $r \in \mathbb{N}$. Then $\tilde{G} \in L^{2}$.

$$
\text { Proof: Then (31) and (29) imply }\|\tilde{G}\|_{2}^{2} \leq r\|\tilde{G}\|_{\infty} / h \text {. }
$$




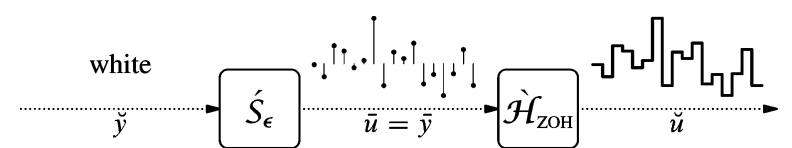

Fig. 8. A periodic stationary output $\breve{u}$.

In particular every hold and sampler that is in $L^{\infty}$ is necessarily in $L^{2}$.

The $L^{2}$ system norm defined by (31) retains familiar deterministic and stochastic interpretations. For SISO $h$-shift-invariant analog systems, for instance, the norm satisfies [26]

$$
\|\breve{G}\|_{2}^{2}=\frac{1}{h} \int_{0}^{h}\|\mathcal{G} \delta(\cdot-\sigma)\|_{L^{2}(\mathbb{R})}^{2} d \sigma .
$$

That is, $\|\breve{G}\|_{2}^{2}$ is the average energy of the output where the average is taken over all delta functions applied at $\sigma \in[0, h)$. For $h \downarrow 0$ this reduces to the classic LTI result. Also, stochastic interpretations are maintained: $\|\breve{G}\|_{2}^{2}$ equals the over time averaged sum of variances (power) of the output elements if the system is driven by standard white noise [26].

Example 5.3: Consider again the HSP $\mathcal{H}_{\mathrm{ZOH}} \mathcal{S}_{\epsilon}$ studied in Example 5.2. As the input $y$ to this system ranges over the delta functions applied at $\sigma \in[0, h)$ the output of the sampler ranges over $\bar{y} \equiv 0$ for $\sigma \in[0, h-\epsilon]$ and $\bar{y}[i]=(1 / \epsilon) \bar{\delta}[i-1]$ for $\sigma \in[h-\epsilon, h)$. Hence for $\sigma \in[0, h-\epsilon]$ the output energy of the hold is zero while for $\sigma \in[h-\epsilon, h)$ the output energy is $\left\|(1 / \epsilon) \mathbb{1}_{[h, 2 h]}\right\|_{2}^{2}=\left(h / \epsilon^{2}\right)$. The average energy therefore equals

$$
\left\|\grave{\mathcal{H}}_{\mathrm{ZOH}} \dot{\mathcal{S}}_{\epsilon}\right\|_{2}^{2}=\frac{1}{h} \int_{h-\epsilon}^{h} \frac{h}{\epsilon^{2}} \mathrm{~d} \tau=\frac{1}{\epsilon} .
$$

The cascade of the ideal sampler and the zero-order hold consequently has infinite $L^{2}$ system norm.

When driven by zero mean unit intensity white noise $\breve{y}$, the samples $\bar{u}=\bar{y}=\dot{\mathcal{S}}_{\epsilon} \breve{y}$ for this sampler are independent and are stationary with variance $1 / \epsilon$. The "Manhattan skyline" output $\breve{u}=\grave{\mathcal{H}}_{\mathrm{ZOH}} \bar{u}$ shown in of Fig. 8 clearly is not stationary as an analog signal because it is piecewise constant, but it is stationary as a lifted signal. Its over time averaged power is well defined and equals $\left\|\grave{\mathcal{H}}_{\mathrm{ZOH}} \dot{\mathcal{S}}_{\epsilon}\right\|_{2}^{2}=(1 / \epsilon)$.

Signal connotations are not that consistent in semilifted cases, where deterministic and stochastic interpretations might require different scaling. To be specific, to maintain the deterministic interpretation for A/D systems (averaging the output energy over all $\delta$-functions applied in $[0, h)$ ), we still need to scale the Hilbert-Schmidt norm by a factor of $1 / h$. At the same time, this factor is not required to maintain the stochastic interpretation (the response to the analog white noise is a stationary discrete process then). D/A systems, on the contrary, do not need the scaling in the deterministic case, whereas do need it to maintain the stochastic meaning. We nevertheless proceed with the scaling in all cases of interest, just to keep the exposition simple.
The $L^{2}$ system norm (31) corresponds to the system inner product

$$
\langle\tilde{G}, \tilde{P}\rangle_{2}=\frac{1}{2 \pi h} \int_{-\pi}^{\pi}\left\langle\tilde{G}\left(\mathrm{e}^{\mathrm{j} \theta}\right), \tilde{P}\left(\mathrm{e}^{\mathrm{j} \theta}\right)\right\rangle_{\mathrm{HS}} \mathrm{d} \theta
$$

with the Hilbert-Schmidt inner product defined as

$$
\operatorname{tr}\left(A B^{*}\right)=\operatorname{tr}\left(B^{*} A\right):=\langle A, B\rangle_{\mathrm{HS}}:=\sum_{i}\left\langle A e_{i}, B e_{i}\right\rangle_{\mathbb{K}_{\mathrm{o}}}
$$

where $\left\{e_{i}\right\}$ is any complete orthonormal sequence of $\mathbb{K}_{i}$. By Parseval's theorem the inner product (32) equals

$$
\langle\tilde{G}, \tilde{P}\rangle_{2}=\frac{1}{h} \sum_{k \in \mathbb{Z}}\langle\tilde{G}[k], \tilde{P}[k]\rangle_{\mathrm{HS}}
$$

where $\tilde{G}[k]$ is the impulse response kernel [cf. (10), (12), (13)]. It implies that two $L^{2}$ systems are orthogonal if their impulse response kernels have disjoint supports and that

$$
\|\tilde{G}\|_{2}^{2}=\frac{1}{h} \sum_{k \in \mathbb{Z}}\|\tilde{G}[k]\|_{\mathrm{HS}}^{2} \cdot
$$

This expression is quite useful in various applications.

Finally a note on adjoints. We take adjoints of systems (operators) always with respect to the standard $L^{2}$ and $\ell^{2}$ signal inner product (25). The reason is that these are also adjoints for the other inner products such as (32). A further useful fact is that the system inner product (32) inherits from the Hilbert-Schmidt inner product the trace-like property that

$$
\langle\tilde{A}, \tilde{B} \tilde{X}\rangle_{2}=\left\langle\tilde{A} \tilde{X}^{*}, \tilde{B}\right\rangle_{2}
$$

if $\tilde{X} \in L^{\infty}$ and $\tilde{A}, \tilde{B} \in L^{2}$.

\section{Stability AND CAUSALITY}

This section reviews the notions of stability and causality and their expression in the lifted frequency domain.

\section{A. System Stability}

As HSPs, like that in Fig. 1, typically operate in open loop and their components are implemented separately, we require that each component, i.e., $\mathcal{S}, \overline{\mathcal{F}}$, and $\mathcal{H}$, is stable. We say that $\mathcal{S}$ is stable if it is a bounded operator $L^{2}(\mathbb{R}) \rightarrow \ell^{2}(\mathbb{Z}), \overline{\mathcal{F}}$ is stable if it is a bounded operator $\ell^{2}(\mathbb{Z}) \rightarrow \ell^{2}(\mathbb{Z})$, and $\mathcal{H}$ is stable if it is a bounded operator $\ell^{2}(\mathbb{Z}) \rightarrow L^{2}(\mathbb{R})$. Obviously, in the lifted domain, for the lifted HSP in Fig. 6, all these definitions read as the boundedness as an operator $\ell^{2}(\mathbb{Z}) \rightarrow \ell^{2}(\mathbb{Z})$.

The fact that all components of the lifted HSP are LTI makes it possible to verify their stability to the (lifted) frequency domain. Indeed, because the Fourier transform is an isomorphism from $\ell^{2}(\mathbb{Z})$ to $L^{2}(\mathbb{T})$, each of the systems $\mathcal{S}, \overline{\mathcal{F}}$, and $\mathcal{H}$ is stable iff its lifted transfer function is a bounded operator $L^{2}(\mathbb{T}) \rightarrow L^{2}(\mathbb{T})$. The following result, which is essentially the first part of $[14$, Thm. A.6.26], plays then a key role.

Theorem 6.1: The set of all bounded multiplication operators from $L^{2}(\mathbb{T})$ to $L^{2}(\mathbb{T})$ is $L^{\infty}$. Moreover, the induced norm of an operator $\tilde{O}: L^{2}(\mathbb{T}) \rightarrow L^{2}(\mathbb{T})$ is $\|\tilde{O}\|_{\infty}$. 
It follows from Theorem 6.1 that a sampler $\mathcal{S}$ is stable iff its lifted transfer function $S(z) \in L^{\infty}$ and a hold $\mathcal{H}$ is stable iff its lifted transfer function $\dot{H}(z) \in L^{\infty}$. Propositions 5.1 and 5.2 reduce the verification of these conditions to matrix (or even scalar) operations. For example, $\mathcal{S}$ is stable iff each row of the lifted Fourier transform of its sampling function $\psi(t)$ belongs to $\mathbb{L}$ for (almost) all $\theta$ or, alternatively, iff the magnitude of the Fourier transform of each entry of $\psi(t)$ is square summable over all aliased frequencies for (almost) all baseband frequencies. The latter condition is guaranteed if the Fourier transform of the sampling function decays faster than $1 / \sqrt{\omega}$ as $\omega \rightarrow \infty$, which agrees with known results about stability of the sampling operation [27].

\section{B. Systems Causality}

The notion of causality is well understood for both analog and discrete systems. Intuitively, a system is causal if its output at any time instance depends only upon its past and present inputs and does not depend on the future inputs. For a continuous-time system $\mathcal{G}$ this can be formally expressed as

$$
\Pi_{T} \mathcal{G}\left(I-\Pi_{T}\right)=0, \quad \forall T \in \mathbb{R}
$$

where the truncation operator $\Pi_{T}$ is defined via the relation

$$
\left(\Pi_{T} u\right)(t)= \begin{cases}u(t) & t<T \\ 0 & t \geq T\end{cases}
$$

The discrete-time case is the same modulo the use of the discrete truncation operator $\bar{\Pi}_{k}$, defined similarly. If the system is time invariant, the condition need only be checked for one fixed $T$, e.g., for $T=0$.

The extension of these notions to hybrid systems depends on the way in which continuous and discrete times are synchronized. Henceforth, motivated mainly by the time association in the lifting transformation, we presume that the $k$ th discrete instance corresponds to the whole continuous-time interval $[k h,(k+1) h)$. In this case, we say that a (shift-invariant) sampler $\mathcal{S}$ is causal if

$$
\bar{\Pi}_{k} \mathcal{S}\left(I-\Pi_{k h}\right)=0, \text { for some } k \in \mathbb{Z}
$$

and a (shift-invariant) hold $\mathcal{H}$ is causal if

$$
\Pi_{k h} \mathcal{H}\left(I-\bar{\Pi}_{k}\right)=0 \text {, for some } k \in \mathbb{Z} \text {. }
$$

It can be verified that, according to these definitions, sampler (3) is causal iff $\psi(t)=0$ for all $t \leq-h$ and hold (4) is causal iff $\phi(t)=0$ for all $t<0$. While the latter is in agreement with the criterion for continuous-time systems, the former might appear peculiar. For example, a sampler with the sampling function $\psi(t+h / 2)$, which acts as $\bar{y}[k]=y(k h+h / 2)$, is causal by this definition. This, however, is a matter of convention. If the implementation permits $\bar{y}[k]$ to depend only upon $y(t)$ for $t<k h$, we may require from $\mathcal{S}$ to be strictly causal, i.e., that $\bar{\Pi}_{k+1} \mathcal{S}\left(I-\Pi_{k h}\right)=0$.

Definitions (36) and (37) can be lifted straightforwardly. To this end, note that $\Pi_{k h}$ corresponds to

$$
\left(\breve{\Pi}_{k} \breve{u}\right)[i]= \begin{cases}\breve{u}[i] & i<k \\ 0 & i \geq k\end{cases}
$$

in the lifted domain. Thus, both (36) and (37) became particular cases of the general definition: an LTI (discrete/semi-lifted/ lifted) system $\tilde{\mathcal{G}}$ is causal if

$$
\tilde{\Pi}_{k} \tilde{\mathcal{G}}\left(I-\tilde{\Pi}_{k}\right)=0, \text { for some } k \in \mathbb{Z} .
$$

Remark 6.1: When applied to the lifting $\breve{\mathcal{G}}$ of a continuoustime system $\mathcal{G}$, definition (38) reads $\Pi_{k h} \mathcal{G}\left(I-\Pi_{k h}\right)=0$. This is not equivalent to (35), unless $\mathcal{G}$ is time invariant. Much care must therefore be taken in analyzing causality in the lifted domain with this definition. Throughout, we use the lifted version of (38) only in relation to lifted HSP blocks, in which case it does reflect causality (with the convention about the sampler discussed above).

We also need a more general definition. We say that an LTI system $\tilde{\mathcal{G}}$ is $l$-causal $(l \in \mathbb{Z})$ if

$$
\tilde{\Pi}_{k-l} \tilde{\mathcal{G}}\left(I-\tilde{\Pi}_{k}\right)=0, \text { for some } k \in \mathbb{Z} .
$$

This definition allows the output of $\tilde{\mathcal{G}}$ at the moment $k$ to depend on its input at all moments $\leq k+l$. If $l>0$, this effectively says that $\tilde{\mathcal{G}}$ may have $l$ steps preview. If $l<0,(39)$ defines a system with the delay of $-l$. The case of $l=-1$ corresponds to strictly causal systems.

\section{Stability With Causality Constraints}

Our message in this subsection is that $(l)$ causality can be neatly incorporated into the stability analysis, in both time and frequency domains.

Let $\tilde{\mathcal{G}}$ be a stable, i.e., bounded mapping $\ell^{2}(\mathbb{Z}) \rightarrow \ell^{2}(\mathbb{Z})$, (discrete/semi-lifted/lifted) system and consider (39) for $k=0$. It is readily seen that $\tilde{\Pi}_{-l}$ and $I-\tilde{\Pi}_{0}$ are the orthogonal projections from $\ell^{2}(\mathbb{Z})$ to $\ell^{2}\left(\mathbb{Z}_{-l}^{-}\right)$and $\ell^{2}\left(\mathbb{Z}_{0}^{+}\right)$, respectively. Thus, (39) reads $\tilde{\Pi}_{-l} \tilde{\mathcal{G}} \ell^{2}\left(\mathbb{Z}_{0}^{+}\right)=0$ or, equivalently

$$
\tilde{\mathcal{G}} \ell^{2}\left(\mathbb{Z}_{0}^{+}\right) \subset \ell^{2}(\mathbb{Z}) \ominus \ell^{2}\left(\mathbb{Z}_{-l}^{-}\right)=\ell^{2}\left(\mathbb{Z}_{-l}^{+}\right) .
$$

Thus, we just showed that an LTI system $\tilde{\mathcal{G}}$ is stable and $l$-causal iff it is a bounded operator $\ell^{2}\left(\mathbb{Z}_{0}^{+}\right) \rightarrow \ell^{2}\left(\mathbb{Z}_{-l}^{+}\right)$.

Because the $z$-transform is an isometric isomorphism between $\ell^{2}\left(\mathbb{Z}_{l}^{+}\right)$and $z^{l} H^{2}$, the stability condition above can be reformulated as follows: $\tilde{\mathcal{G}}$ is stable and causal iff its transfer function $\tilde{G}(z)$ is a bounded operator $H^{2} \rightarrow z^{l} H^{2}$. This, in turn, translates to (relatively) easily verifiable properties of $\tilde{G}(z)$ with the help of the following result.

Theorem 6.2: The set of all bounded multiplication operators from $H^{2}$ to $z^{l} H^{2}$ is $z^{l} H^{\infty}$. Moreover, the induced norm of an operator $\tilde{O}: H^{2} \rightarrow z^{l} H^{2}$ is $\|\tilde{O}\|_{\infty}$.

Proof: The result for $l=0$ (i.e., for the causal case) is known, see [14, Thm.A.6.26]. To extend it to general $l$, note that according to the definition of $z^{l} H^{2}$

$$
\tilde{O} H^{2} \subset z^{l} H^{2} \Leftrightarrow z^{-l}\left(\tilde{O} H^{2}\right) \subset H^{2} \Leftrightarrow\left(z^{-l} \tilde{O}\right) H^{2} \subset H^{2} .
$$

According to the result for $l=0$, the latter reads $z^{-l} \tilde{O} \in H^{\infty}$, leading to the first part. The second part follows by the fact that the multiplication by $z^{-l}$ does not alter the $L^{\infty}$-norm.

It follows from Theorem 6.2 that $\mathcal{S}$ and $\mathcal{H}$ are stable and $l$-causal iff their lifted transfer functions, $S(z)$ and $\grave{H}(z)$, re- 
spectively, belong to $z^{l} H^{\infty}$. Thus, if causality constraints are incorporated into an optimization procedure, it is no longer sufficient to look at frequency responses (transfer functions at $z \in$ $\mathbb{T})$. The behavior of transfer functions at the whole region of $z \in \mathbb{C} \backslash \mathbb{D}$ should be accounted for. This complicates the analysis and design considerably. As is known from the causal Wiener filtering, the design then involves some form of spectral factorization and while, admittedly, this complicates matters, it is the point of [28, Part III] that the machinery of this paper can indeed be put to use in solving the optimal design problem with respect to holds of given degree of causality.

\section{CONCLUDING REMARKS}

In this paper we have collected the basic concepts of the system-theoretic approach to sampled signal reconstruction and technical material of lifting and lifted signals and systems, in both time and frequency domains. The key point is that lifting may losslessly recover time-invariance (in discrete time) of systems that are not time-invariant in continuous time. From that point on most of the results are intuitively clear, albeit possibly technically advanced, and follow corresponding results of standard discrete systems. It is this material that forms the basis for the solutions to the optimal noncausal signal reconstruction problems considered in Part II, [17], and for the optimal relaxedly causal reconstruction problems considered in [28, Part III].

\section{ACKNOWLEDGMENT}

The authors would like to thank anonymous reviewers for their valuable comments and suggestions that were helpful in improving the paper.

\section{REFERENCES}

[1] A. J. Jerri, "The Shannon sampling theorem-Its various extensions and applications: A tutorial review," Proc. IEEE, vol. 65, pp. 1565-1596, 1977.

[2] M. Unser, "Sampling-50 years after Shannon," Proc. IEEE, vol. 88, pp. 569-587, 2000.

[3] P. P. Vaidyanathan, "Generalizations of the sampling theorem: Seven decades after Nyquist," IEEE Trans. Circuits Syst. I, vol. 48, pp. 1094-1109, 2001.

[4] A. Aldroubi and K. Gröchenig, "Nonuniform sampling and reconstruction in shift-invariant spaces," SIAM Rev., vol. 43, no. 4, pp. 585-620, 2001.

[5] J. R. Higgins, Sampling Theory in Fourier and Signal Analysis: Foundations. Oxford, U.K.: Oxford Univ. Press, 1996.

[6] Y. C. Eldar and M. Unser, "Nonideal sampling and interpolation from noisy observations in shift-invariant spaces," IEEE Trans. Signal Process., vol. 54, pp. 2636-2651, 2006.

[7] T. Chen and B. A. Francis, Optimal Sampled-Data Control Systems. London, U.K.: Springer-Verlag, 1995.

[8] G. E. Dullerud, Control of Uncertain Sampled-Data Systems. Boston, MA: Birkhäuser, 1996.

[9] P. P. Khargonekar and Y. Yamamoto, "Delayed signal reconstruction using sampled-data control," in Proc. 35th IEEE Conf. Decision Contr., Kobe, Japan, 1996, pp. 1259-1263.

[10] H. M. Robbins, "An extension of Wiener filter theory to partly sampled systems," IRE Trans. Circuit Theory, vol. CT-6, pp. 362-370, 1959.
[11] T. Chen and B. A. Francis, "Design of multirate filter banks by $H^{\infty}$ optimization," IEEE Trans. Signal Process., vol. 43, pp. 2822-2830, 1995.

[12] J. G. Proakis and D. G. Manolakis, Digital Signal Processing, 4th ed Upper Saddle River, NJ: Prentice-Hall, 2007.

[13] J. B. Thomas, An Introduction to Statistical Communication Theory. New York: Wiley, 1969.

[14] R. F. Curtain and H. Zwart, An Introduction to Infinite-Dimensional Linear Systems Theory. New York: Springer-Verlag, 1995.

[15] T. Kailath, A. H. Sayed, and B. Hassibi, Linear Estimation. Upper Saddle River, NJ: Prentice-Hall, 2000.

[16] C. Scherer, "Mixed $\mathrm{H}_{2} / \mathrm{H}_{\infty}$ control," in Trends in Control: A European Perspective, A. Isidori, Ed. Berlin: Springer-Verlag, 1995, pp. 173-216.

[17] G. Meinsma and L. Mirkin, "Sampling from a system-theoretic viewpoint: Part II-Non-causal solutions," IEEE Trans. Signal Process., vol. 58,2010 , to be published.

[18] E. I. Jury, Sampled-Data Control Systems. New York: Wiley, 1958.

[19] A. Janssen, "The Zak transform: A signal transform for sampled timecontinuous signals," Philips J. Res., vol. 43, pp. 23-69, 1988.

[20] W. Rudin, Real and Complex Analysis, 3rd ed. New York: McGrawHill, 1987.

[21] J. Braslavsky, G. Meinsma, R. Middleton, and J. Freudenberg, "On a key sampling formula relating the Laplace and $\mathcal{Z}$ transforms," Syst. Contr. Lett., vol. 29, no. 4, pp. 181-190, 1997.

[22] A. V. Oppenheim, A. S. Willsky, and S. H. Nawab, Signals and Systems, 2nd ed. Upper Saddle River, NJ: Prentice-Hall, 1997.

[23] K. J.Åström and B. Wittenmark, Computer-Controlled Systems: Theory and Design, 3rd ed. Englewood Cliffs, NJ: Prentice-Hall, 1997.

[24] B. Bamieh and J. B. Pearson, "A general framework for linear periodic systems with applications to $H^{\infty}$ sampled-data control," IEEE Trans. Autom. Control, vol. 37, no. 4, pp. 418-435, 1992.

[25] Y. Yamamoto and P. P. Khargonekar, "Frequency response of sampleddata systems," IEEE Trans. Autom. Control, vol. 41, no. 2, pp. 166-176, 1996.

[26] B. Bamieh and J. B. Pearson, "The $\mathcal{H}^{2}$ problem for sampled-data systems," Syst. Contr. Lett., vol. 19, no. 1, pp. 1-12, 1992.

[27] Y. Kannai and G. Weiss, "Approximating signals by fast impulse sampling," Math. Contr., Signals Syst., vol. 6, pp. 166-179, 1993.

[28] G. Meinsma and L. Mirkin, Sampling From a System-Theoretic Viewpoint, Dept. Appl. Math., Univ. Twente, 2009 [Online]. Available: http://eprints.eemcs.utwente.n1/16463/

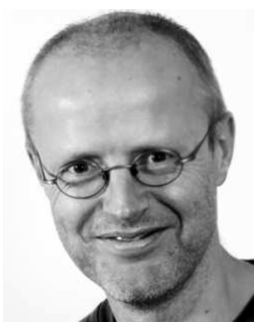

Gjerrit Meinsma was born in Opeinde, The Netherlands, in 1965. He received the Ph.D. degree from the University of Twente in 1993.

During the following three years, he held a Postdoctoral position with the University of Newcastle, Australia. Since 1997, he has been with the Department of Applied Mathematics, University of Twente, The Netherlands. His research interests are in mathematical systems and control theory, in particular robust control theory.

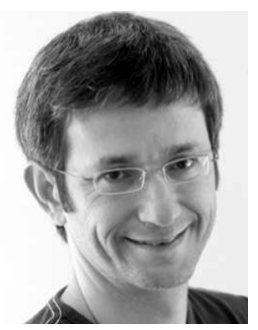

Leonid Mirkin (M'99) was born in Frunze, Kirghiz SSR, USSR (now Bishkek, Kyrgyz Republic). He received the electrical engineer degree from Frunze Polytechnic Institute and the Ph.D. (candidate of sciences) degree in automatic control from the Institute of Automation, Academy of Sciences of Kyrgyz Republic, in 1989 and 1992, respectively.

From 1989 to 1993 , he was with the Institute of Automation, Academy of Sciences of Kyrgyz Republic. In 1994, he joined the Faculty of Mechanical Engineering, Technion-Israel Institute of Technology, first as a Postdoctoral Researcher and then as a faculty member. His research interests include systems theory, control, and estimation of sampled-data systems, dead-time compensation, systems with preview, the application of control to electromechanical and optical devices, and robustifying properties of corruption. 